\title{
A search for axion-like particles in light-by-light scattering at the CLIC
}

\section{S.C. Inan ${ }^{a}$ and A.V. Kisselev ${ }^{b}$}

\author{
${ }^{a}$ Department of Physics, Sivas Cumhuriyet University, \\ 58140 Sivas, Turkey \\ ${ }^{b}$ Division of Theoretical Physics, A.A. Logunov Institute for High Energy Physics, \\ NRC "Kurchatov Institute", 142281 Protvino, Russian Federation \\ E-mail: sceminan@cumhuriyet.tr, alexandre.kisselev@ihep.ru
}

\begin{abstract}
The virtual production of axion-like particles (ALPs) in the light-by-light scattering at the CLIC collider is studied. Both differential and total cross sections are calculated, assuming interaction of the ALP with photons via CP-odd term in the Lagrangian. The $95 \%$ C.L. exclusion regions for the ALP mass and its coupling constant are given. By comparing our results with existing collider bounds, we see that the ALP search at the CLIC has a great physics potential of searching for the ALPs, especially, in the mass region $1 \mathrm{TeV}-2.4 \mathrm{TeV}$, with the collision energy $\sqrt{s}=3000 \mathrm{GeV}$ and integrated luminosity $L=5000 \mathrm{fb}^{-1}$ for the Compton backscattered initial photons. In particular, our limits are stronger than recently obtained bounds for the ALP production in the light-by-light scattering at the LHC.
\end{abstract}

KeYwords: Phenomenological Models

ARXIV EPRINT: 2003.01978 


\section{Contents}

1 Introduction 1

2 Light-by-light virtual production of ALP 3

2.1 Compton backscattered photons 6

$\begin{array}{lll}2.2 & \text { Weizsäcker-Williams photons } & 12\end{array}$

$\begin{array}{lll}3 & \text { Conclusions } & 14\end{array}$

A Dependence of total cross section on parameters of axion-like particle

\section{Introduction}

The notion of the QCD axion is closely related to the strong CP problem, which means the absence of the $\mathrm{CP}$ violation in the strong interactions. In its turn, the $\mathrm{CP}$ problem arises as a possible solution to the $\mathrm{U}(1)$ problem. The QCD Lagrangian in the limit of vanishing masses of $u$ and $d$ quarks has a global symmetry $\mathrm{U}(2)_{V} \times \mathrm{U}(2)_{A}=\mathrm{SU}(2)_{I} \times$ $\mathrm{U}(1)_{Y} \times \mathrm{SU}(2)_{A} \times \mathrm{U}(1)_{A}$. The non-zero quark condensates $\langle\bar{u} u\rangle$ and $\langle\bar{d} d\rangle$ break down the axial symmetry $\mathrm{SU}(2)_{A} \times \mathrm{U}(1)_{A}$ spontaneously. As a result, four Nambu-Goldstone bosons should appear. But besides light pions, no another light state is present in the hadronic spectrum since $m_{\eta} \gg m_{\pi}$. It is called the $\mathrm{U}(1)$ problem [1].

The $\mathrm{U}(1)_{A}$ symmetry is connected with a transformation of the fermion fields $\psi \rightarrow$ $e^{i \alpha \gamma_{5}} \psi, \bar{\psi} \rightarrow \bar{\psi} e^{i \alpha \gamma_{5}}$. One possible resolution of the $\mathrm{U}(1)$ problem is provided by the AdlerBell-Jackiw chiral anomaly for the axial current $J_{\mu}^{5}=\bar{\psi} \gamma_{\mu} \gamma_{5} \psi[2,3]$.

Although the axial anomaly is a total divergency, $\mathrm{U}(1)_{A}$ is not a symmetry of the strong interaction for gluon fields $A_{\mu}$ which are pure gauges at spatial infinity $[4,5]$. They are classified by the integer $n, A_{\mu}=(-i / g) \partial_{\mu} \omega \omega^{-1}$, where $\omega \rightarrow \omega_{n}=\exp (i 2 \pi n)$ as $r \rightarrow \infty$. This condition is a definition of a classical vacuum of the gauge field $|n\rangle$. The true or $\theta$-vacuum becomes a superposition of the vacua $|n\rangle,|\theta\rangle=\sum_{n} \exp (-i n \theta)|n\rangle[6]$.

As a result, an effective QCD action acquires so-called $\theta$-term. It breaks $\mathrm{P}$ - ant Tinvariance but conserves $\mathrm{C}$-invariance, so CP-invariance is violated. Thus, it contributes to the neutron electric dipole moment $d_{n}$. The current experimental limit $d_{n}<0.021 \times 10^{-23}$ e cm [7] requires $\theta$ to be less than $10^{-9}$. The smallness of the angle $\theta$ is known as strong CP problem.

The elegant solution of the CP mystery of the SM is provided by the Peccei-Quinn (PQ) mechanism with a new, spontaneously broken approximate global $\mathrm{U}(1)_{\mathrm{PQ}}$ symmetry [8]. As it is shown in $[9,10]$ it leads to a light neutral pseudoscalar particle, the axion a, which is the Nambu-Goldstone boson of the broken $\mathrm{U}(1)_{\mathrm{PQ}}$ symmetry. The idea is to replace the CP-violating term $\theta$ by the CP-conserving axion. Namely, the axion field can be 
redefined to absorb the parameter $\theta$. In fact, the axion replaces the QCD theta parameter by a dynamical quantity, thereby explaining of non-observation of the strong $\mathrm{CP}$ violation. Thus, the PQ mechanism is a compelling solution to the strong CP problem.

In the PQWW scheme [8]-[10] an extra Higgs doublet is used, and the axion mass is related to the electroweak symmetry breaking scale. There are two models in which the PQ symmetry is decoupled from the electroweak (EW) scale and is spontaneously broken. It results in axions with extremely weak couplings ("invisible" axion). One of the models is the KSVZ model $[11,12]$ with one Higgs doublet in which the axion is introduced as the phase of an EW singlet scalar field. This scalar is coupled to an additional heavy quark, and its coupling is induced by the interaction of the heavy quarks with other fields. In the DFSZ model $[13,14]$ two Higgs doublets are used, as well as an additional EW singlet scalar. The latter is coupled to the SM fields through its interaction with the Higgs doublets.

The axion also appears in the context of the string theory [15]-[17]. In the string theory, spin-zero particles must couple to a photon field since all couplings are defined by the expectation value of scalar fields. This implies the existence of the P-odd term in the Lagrangian proportional to

$$
-\frac{1}{4} g_{a \gamma \gamma} a F_{\mu \nu} \tilde{F}^{\mu \nu}=g_{a \gamma \gamma} a \vec{E} \cdot \vec{B}
$$

where $F_{\mu \nu}$ is the electromagnetic tensor, $\tilde{F}_{\mu \nu}=(1 / 2) \varepsilon_{\mu \nu \rho \sigma} F^{\rho \sigma}$ its dual, and $a$ is the QCD axion or axion-like particle (ALP) [18]. ALPs can also appear in theories with spontaneously broken symmetries $[19,20]$ or in GUT [21]. Lately, a number of new theoretical schemes with the axion as a basic quantity was developed [23]-[30]. For a review on the axions and ALPs, see [31]-[34] and references therein.

Both theory and phenomenology of the axions were also studied in large [35]-[38] and warped [39]-[41] extra dimensions (EDs). In an ED framework, the mass of the axion becomes independent of the scale associated with the breaking of the PQ symmetry. It means that the axion mass can be treated independently of its couplings to the SM fields.

The very low mass and small coupling axion and/or ALP are a leading dark matter (DM) candidate, since their properties, allow them to be stable and difficult-to-detect. Both axions and ALPs can be produced in the early Universe and therefore constitute most of the cold DM in the Universe [42]-[44] (see also recent papers [45]-[52]). The relevance of the QCD axion and, more generally, of ALPs in astrophysics and cosmology is of particular interest [53]-[58]. Many axion DM experiments are in progress [59]-[67] (see also [68]).

The axion phenomenology involves phenomena such as stellar evolution, axion mediated forces, dark matter detection, axion decays, axion-photon conversion, so-called "light shining trough the wall", etc.

There is a broad experimental program aiming to search for the QCD axion via its coupling to the SM. On the other hand, many ALP searches assume their strong couplings to the electromagnetic term $F_{\mu \nu} \tilde{F}^{\mu \nu}$ as in eq. (1.1). In terrestrial experiments, bounds on very low mass axions and small mass axions were obtained [69]-[78]. The coupling of the ALPs to other gauge bosons is also studied (see, for instance, [79]). Note that the ALPs are not directly relevant for the QCD axion. Therefore, heavy ALPs can be detected at 
colliders, in particular, in a light-by-light scattering [80]-[85]. As it was shown in [86], searches at the LHC with the use of the proton tagging technique can constrain the ALP masses in the region $0.5 \mathrm{TeV}-2 \mathrm{TeV}$.

Compact Linear Collider (CLIC) is the linear collider that is planned to accelerate and collide electrons and positrons at maximally $3 \mathrm{TeV}$ center-of-mass energy [87]. In the CLIC, it is possible to obtain accelerating gradients of $100 \mathrm{MV} / \mathrm{m}$. Three energy states are considered to operate CLIC at maximum efficiency [88]. The $\sqrt{s}=380 \mathrm{GeV}$ is the first one and it is possible to reach the integrated luminosity $L=1000 \mathrm{fb}^{-1}$. This energy stage cover Higgs boson, top quark, and gauge sectors. It is planned to examine such SM particles with high precision [89]. The second one has $\sqrt{s}=1500 \mathrm{GeV}$ center-of-mass energy and $2500 \mathrm{fb}^{-1}$ integrated luminosity. At this stage, it is enabled to investigate beyond the SM physics. Also, a detailed analysis of the Higgs boson can be made, such as the Higgs selfcoupling, the top-Yukawa coupling, and rare Higgs decay channels. [90]. The third stage of the CLIC has a maximum center-of-mass energy value $\sqrt{s}=3000 \mathrm{GeV}$ and integrated luminosity value $L=5000 \mathrm{fb}^{-1}$. At this stage, the most precise examinations of the $\mathrm{SM}$ is possible. Moreover, it is enabled to discover beyond the SM heavy particles of mass greater than $1500 \mathrm{GeV}$ [89]. The new physics search potential of the CLIC is presented in [91, 92].

At the CLIC, it is possible to study $\gamma \gamma$ and $e \gamma$ collider with real photons. These $\gamma$ beams are gotten by the Compton backscattering of laser photons off linear electron beams. Another options for the $\gamma \gamma$ and $e \gamma$ collisions are photon-induced processes at the CLIC. In this type of process, the photons are emitted from the incoming electron beams. The photons scatter at tiny angles from the beam pipe. Hence, they have very low virtuality; that is why these photons are called "almost-real".

The first evidence of the subprocess $\gamma \gamma \rightarrow \gamma \gamma$ was observed by the ATLAS Collaboration in high-energy ultra-peripheral $\mathrm{PbPb}$ collisions [93]. The same process was also reported by the CMS Collaboration [94]. Recently, the ATLAS Collaboration have published the evidence of the light-by-light scattering with the certainty of 8,2 sigma [95]. The analysis of the exclusive and diffractive $\gamma \gamma$ production in $\mathrm{PbPb}$ collisions was done in $[96,97]$. We have examined a possibility to constrain the parameters of the model with a warped ED in the photon-induced process $p p \rightarrow p \gamma \gamma p \rightarrow p^{\prime} \gamma \gamma p^{\prime}$ at the LHC [98]. Previously, the photon-induced processes in EDs were studied in [99, 100].

In the present paper, we propose to search for the ALP $a$ in the exclusive light-by-light scattering at the lepton collider CLIC.

In the next section differential and total cross sections are calculated as functions of the ALP mass $m_{a}$ and its coupling $f$. It enables us to estimate the CLIC exclusion regions for both types of the initial photons.

\section{Light-by-light virtual production of ALP}

The pseudoscalar ALP couples to the SM photons via

$$
\mathcal{L}_{a}=\frac{1}{2}\left(\partial_{\mu} a\right)\left(\partial^{\mu} a\right)-\frac{1}{2} m_{a}^{2} a^{2}+\frac{a}{f_{a}^{(-)}} F_{\mu \nu} F^{\mu \nu}+\frac{a}{f_{a}^{(+)}} F_{\mu \nu} \tilde{F}^{\mu \nu},
$$


were $\left(f_{a}^{(-)}\right)^{-1}$ and $\left(f_{a}^{(+)}\right)^{-1}$ are the ALP-photon couplings in CP-odd and CP-even terms of the interaction Lagrangian. Note that, in contrast to the true QCD axion, the mass and couplings of the ALP are independent parameters. In what follows, we assume that only the last term is realized in $(2.1)$ with $f_{a}^{(+)}=f$. As for a possible contribution from the third term in (2.1), it is discussed in the section Conclusions.

The explicit expressions for the photon spectrum are given below. The differential cross section is the following sum of helicity amplitudes squared [83, 84]

$$
\frac{d \sigma}{d \Omega}=\frac{1}{128 \pi^{2} s}\left(\left|M_{++++}\right|^{2}+\left|M_{+-+-}\right|^{2}+\left|M_{+--+}\right|^{2}+\left|M_{++--}\right|^{2}\right) .
$$

Here and below $s, t$ and $u$ are the Mandelstam variables of the diphoton system. Each of the helicity amplitudes is a sum of the ALP and SM terms,

$$
M=M_{a}+M_{\mathrm{ew}}
$$

The explicit expressions of the pure ALP amplitudes can be found in [83, 84]. In particular,

$$
\begin{aligned}
\mathfrak{R e} M_{++++}^{(a)} & =-\frac{4}{f_{a}^{2}} \frac{s^{2}\left(s-m_{a}^{2}\right)}{\left(s-m_{a}^{2}\right)^{2}+m_{a}^{2} \Gamma_{a}^{2}}, \\
\mathfrak{I m} M_{++++}^{(a)} & =\frac{4}{f_{a}^{2}} \frac{s^{2} m_{a} \Gamma_{a}}{\left(s-m_{a}^{2}\right)^{2}+m_{a}^{2} \Gamma_{a}^{2}},
\end{aligned}
$$

where $\Gamma_{a}$ is the total width of the ALP

$$
\Gamma_{a}=\frac{\Gamma(a \rightarrow \gamma \gamma)}{\operatorname{Br}(a \rightarrow \gamma \gamma)}
$$

and

$$
\Gamma(a \rightarrow \gamma \gamma)=\frac{m_{a}^{3}}{4 \pi f^{2}}
$$

is its decay width into two photons. Correspondingly, we have [83, 84]

$$
\begin{aligned}
\mathfrak{R e} M_{+-+-}^{(a)} & =-\frac{4}{f_{a}^{2}} \frac{u^{2}}{u-m_{a}^{2}}, \quad \mathfrak{I m} M_{+-+-}^{(a)}=0, \\
\mathfrak{R e} M_{+--+}^{(a)} & =-\frac{4}{f_{a}^{2}} \frac{t^{2}}{t-m_{a}^{2}}, \quad \mathfrak{I m} M_{+--+}^{(a)}=0, \\
\mathfrak{R e} M_{++--}^{(a)} & =\frac{4}{f_{a}^{2}}\left(\frac{s^{2}\left(s-m_{a}^{2}\right)}{\left(s-m_{a}^{2}\right)^{2}+m_{a}^{2} \Gamma_{a}^{2}}+\frac{t^{2}}{t-m_{a}^{2}}+\frac{u^{2}}{u-m_{a}^{2}}\right), \\
\mathfrak{I m} M_{++--}^{(a)} & =-\frac{4}{f_{a}^{2}} \frac{s^{2} m_{a} \Gamma_{a}}{\left(s-m_{a}^{2}\right)^{2}+m_{a}^{2} \Gamma_{a}^{2}}, \\
M_{+++-}^{(a)} & =0 .
\end{aligned}
$$

An account of the ALP width $\Gamma_{a}$ is mainly important in a vicinity of the point $s \sim m_{a}^{2}$. That is why, it is omitted in the denominators in eqs. (2.7), (2.8), as well as in the last two terms in the first row of eq. (2.9). 
The SM (electroweak) amplitude is a sum of the fermion and $W$ boson one-loop amplitudes

$$
M_{\mathrm{ew}}=M^{f}+M^{W}
$$

The amplitudes $M_{++++}^{f}(s, t, u)$ and $M_{++++}^{W}(s, t, u)$ are calculated in [101-103] (see also [100])

$$
\begin{aligned}
\frac{1}{\alpha_{\mathrm{em}}^{2} e_{f}^{4}} \mathfrak{R e} M_{++++}^{f}(s, t, u)= & -8-8\left(\frac{u-t}{s}\right) \ln \left(\frac{u}{t}\right) \\
& -4\left(\frac{t^{2}+u^{2}}{s^{2}}\right)\left[\ln ^{2}\left(\frac{u}{t}\right)+\pi^{2}\right], \\
\mathfrak{I m} M_{++++}^{f}(s, t, u)= & 0
\end{aligned}
$$

where $e_{f}$ is the fermion electric charge in units of the proton charge,

$$
\begin{aligned}
\frac{1}{\alpha_{\mathrm{em}}^{2}} \mathfrak{R e} M_{++++}^{W}(s, t, u)= & 12+12\left(\frac{u-t}{s}\right) \ln \left(\frac{u}{t}\right) \\
& +16\left(1-\frac{3 t u}{4 s^{2}}\right)\left[\ln ^{2}\left(\frac{u}{t}\right)+\pi^{2}\right] \\
& +16\left[\frac{s}{t} \ln \left(\frac{s}{m_{W}^{2}}\right) \ln \left(\frac{-t}{m_{W}^{2}}\right)+\frac{s}{u} \ln \left(\frac{s}{m_{W}^{2}}\right) \ln \left(\frac{-u}{m_{W}^{2}}\right)\right. \\
& \left.+\frac{s^{2}}{t u} \ln \left(\frac{-t}{m_{W}^{2}}\right) \ln \left(\frac{-u}{m_{W}^{2}}\right)\right] \\
\frac{1}{\alpha_{\mathrm{em}}^{2}} \mathfrak{I m} M_{++++}^{W}(s, t, u)= & -16 \pi\left[\frac{s}{t} \ln \left(\frac{-t}{m_{W}^{2}}\right)+\frac{s}{u} \ln \left(\frac{-u}{m_{W}^{2}}\right)\right] .
\end{aligned}
$$

The amplitudes $M_{+-+-}^{f, W}(s, t, u)$ and $M_{+--+}^{f, W}(s, t, u)$ can be obtained with the use of the following relations

$$
\begin{aligned}
& M_{+-+-}(s, t, u)=M_{++++}(u, t, s), \\
& M_{+--+}(s, t, u)=M_{++++}(t, s, u)=M_{++++}(t, u, s) .
\end{aligned}
$$

Note that $M_{++++}(s, t, u)=M_{++++}(s, u, t)$, since it depends only on $s$. In particular, we get

$$
\begin{aligned}
\frac{1}{\alpha_{\mathrm{em}}^{2} e_{f}^{4}} \mathfrak{R e} M_{+-+-}^{f}(s, t, u)= & -8-8\left(\frac{s-t}{u}\right) \ln \left(\frac{s}{-t}\right) \\
& -4\left[\left(\frac{t^{2}+s^{2}}{u^{2}}\right) \ln ^{2}\left(\frac{s}{-t}\right)+\pi^{2}\right], \\
\frac{1}{\alpha_{\mathrm{em}}^{2} e_{f}^{4}} \mathfrak{I m} M_{+-+-}^{f}(s, t, u)= & 8 \pi\left[\frac{s-t}{u}+\frac{t^{2}+s^{2}}{u^{2}} \ln \left(\frac{s}{-t}\right)\right],
\end{aligned}
$$


and

$$
\begin{aligned}
\frac{1}{\alpha_{\mathrm{em}}^{2} e_{f}^{4}} \mathfrak{R e} M_{+--+}^{f}(s, t, u)= & -8-8\left(\frac{u-s}{t}\right) \ln \left(\frac{-u}{s}\right) \\
& -4\left[\left(\frac{s^{2}+u^{2}}{t^{2}}\right) \ln ^{2}\left(\frac{-u}{s}\right)+\pi^{2}\right], \\
\frac{1}{\alpha_{\mathrm{em}}^{2} e_{f}^{4}} \mathfrak{I m} M_{+--+}^{f}(s, t, u)= & -8 \pi\left[\frac{u-s}{t}+\frac{s^{2}+u^{2}}{t^{2}} \ln \left(\frac{-u}{s}\right)\right] .
\end{aligned}
$$

The explicit formulas for $M_{+-+-}^{W}(s, t, u)$ have been already derived in [100]

$$
\begin{aligned}
& \frac{1}{\alpha_{\mathrm{em}}^{2}} \mathfrak{R e} M_{+-+-}^{W}(s, t, u)= 12+12\left(\frac{s-t}{u}\right) \ln \left(\frac{s}{-t}\right) \\
&+ 16\left(1-\frac{3 t s}{4 u^{2}}\right) \ln ^{2}\left(\frac{s}{-t}\right) \\
&+16\left[\frac{u}{t} \ln \left(\frac{-u}{m_{W}^{2}}\right) \ln \left(\frac{-t}{m_{W}^{2}}\right)+\frac{u}{s} \ln \left(\frac{-u}{m_{W}^{2}}\right) \ln \left(\frac{s}{m_{W}^{2}}\right)\right. \\
&\left.+\frac{u^{2}}{t s} \ln \left(\frac{-t}{m_{W}^{2}}\right) \ln \left(\frac{s}{m_{W}^{2}}\right)\right] \\
& \frac{1}{\alpha_{\mathrm{em}}^{2}} \mathfrak{I m} M_{+-+-}^{W}(s, t, u)=- {\left[12\left(\frac{s-t}{u}\right)+32\left(1-\frac{3 t s}{4 u^{2}}\right) \ln \left(\frac{s}{-t}\right)\right.} \\
&\left.+16 \frac{u}{s} \ln \left(\frac{-u}{m_{W}^{2}}\right)+16 \frac{u^{2}}{t s} \ln \left(\frac{-t}{m_{W}^{2}}\right)\right] .
\end{aligned}
$$

The explicit expressions for $M_{++--}^{f}(s, t, u)$ and $M_{++--}^{W}(s, t, u)$ are also known [101-103]

$$
\begin{aligned}
& \mathfrak{R e} M_{++--}^{f}(s, t, u)=8 \alpha_{\mathrm{em}}^{2} e_{f}^{4}, \quad \mathfrak{I m} M_{++--}^{f}(s, t, u)=0, \\
& \mathfrak{R e} M_{++--}^{W}(s, t, u)=-12 \alpha_{\mathrm{em}}^{2}, \quad \mathfrak{I m} M_{++--}^{W}(s, t, u)=0 \text {. }
\end{aligned}
$$

Finally, neglecting terms $m_{f}^{2} / s, m_{f}^{2} / t$ and $m_{f}^{2} / u$, we have

$$
\begin{aligned}
& M_{+++-}^{f}(s, t, u) \simeq M_{++--}^{f}(s, t, u), \\
& M_{+++-}^{W}(s, t, u) \simeq M_{++--}^{W}(s, t, u) .
\end{aligned}
$$

\subsection{Compton backscattered photons}

In addition to $e^{+} e^{-}$collisions, $e \gamma$ and $\gamma \gamma$ interactions with real photons can be examined at the CLIC. For this purpose, real photons could be constructed by the Compton backscattering of laser photons off linear electron beam. In this process, a laser photon which has $E_{0}$ energy interacts with an electron. This electron has very high energy $E_{e}$, and it collides with the laser photon at a tiny collision angle $\alpha$ (almost head-on). The scattered photon energy $E_{\gamma}$ can be determined as follows [106-108]

$$
E_{\gamma}=\frac{x_{\max } E_{e}}{1+\left(\theta / \theta_{0}\right)^{2}}, \quad x_{\max }=\frac{\zeta}{\zeta+1}, \quad \theta_{0}=\frac{m_{e}}{E_{e}} \sqrt{\zeta+1}
$$




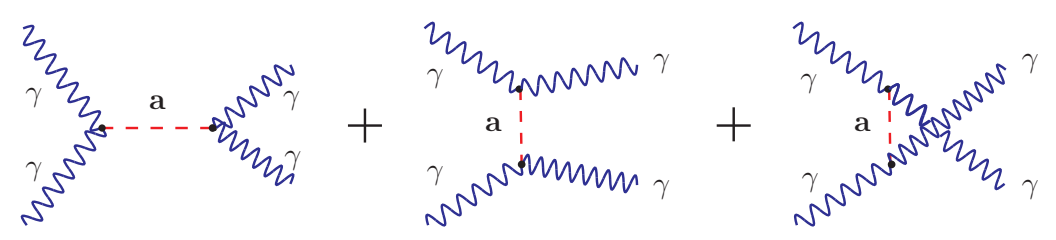

Figure 1. The Feynman diagrams describing light-by-light virtual production of the axion-like particle $a$.

Here $\theta$ is the photon scattering angle from an initial direction of the electron, $x_{\max }=$ $E_{\gamma, \max } / E_{e}$, where $E_{\gamma, \max }$ is the maximum energy of the scattered photons, and

$$
\zeta=\frac{4 E_{e} E_{0}}{m_{e}^{2}}\left[\cos \left(\frac{\alpha}{2}\right)\right]^{2} \simeq \frac{4 E_{e} E_{0}}{m_{e}^{2}}=15.3\left(\frac{E_{e}}{\mathrm{TeV}}\right)\left(\frac{E_{0}}{\mathrm{eV}}\right)
$$

The photon energy $E_{\gamma}$ grows, with an increase of $\zeta$. Hence the energy spectrum gets to be narrower. At the same time, if $\zeta$ value is greater than 4.8 , high energy photons may be lost due to $e^{+} e^{-}$pair creation in interactions of unscattered laser photons with backscattered photons. The invariant energy of the $\gamma \gamma$-system is equal to $s=4 x_{1} x_{2} E_{e}^{2}=$ $4 E_{\gamma_{1}} E_{\gamma_{2}}, x_{i}=E_{\gamma_{i}} / E_{e}$, where $E_{\gamma_{i}}(i=1,2)$ is defined by eqs. (2.21), (2.22).

Most of these real scattered photons have high energy. These Compton backscattered (CB) photons give a spectrum which is defined as follows [104-108]

$$
f_{\gamma / e}(x)=\frac{1}{g(\zeta)}\left[1-x+\frac{1}{1-x}-\frac{4 x}{\zeta(1-x)}+\frac{4 x^{2}}{\zeta^{2}(1-x)^{2}}\right]
$$

where

$$
g(\zeta)=\left(1-\frac{4}{\zeta}-\frac{8}{\zeta^{2}}\right) \log (\zeta+1)+\frac{1}{2}+\frac{8}{\zeta}-\frac{1}{2(\zeta+1)^{2}} .
$$

Note that $x_{\max }$ reaches 0.83 when $\zeta=4.8$. We will use the cut on the final state photon rapidity $\left|\eta_{\gamma \gamma}\right|<2.5$. The cross section of the diphoton production with the CB photon at the CLIC can be found as the integration

$$
d \sigma=2 \int_{z_{\min }}^{z_{\max }} d z z \int_{z^{2} / y_{\max }}^{y_{\max }} \frac{d y}{y} f_{\gamma / e}(y) f_{\gamma / e}\left(z^{2} / y\right) d \sigma(\gamma \gamma \rightarrow \gamma \gamma)
$$

Here

$$
y_{\max }=z_{\max }=0.83, \quad z_{\min }=\frac{p_{\perp}}{E_{e}},
$$

where $p_{\perp}$ is the transverse momentum of the final photons, $f_{\gamma / e}(y)$ is the photon spectrum, and $d \sigma(\gamma \gamma \rightarrow \gamma \gamma)$ is the unpolarized differential cross section of the subprocess $\gamma \gamma \rightarrow \gamma \gamma$. The Feynman diagrams for this process are shown in figure 1. Let us note that in our calculations, we take into account $W$-loop and fermion-loop contributions as the main SM background. The other possible background with fake photons from decays of $\pi^{0}, \eta$, and $\eta^{\prime}$ is negligible in the signal region.

The differential cross sections for the process $\gamma \gamma \rightarrow \gamma \gamma$ for the CB initial photons is shown in figure 2 as functions of the transverse momenta of the final photons $p_{t}$. The ALP 

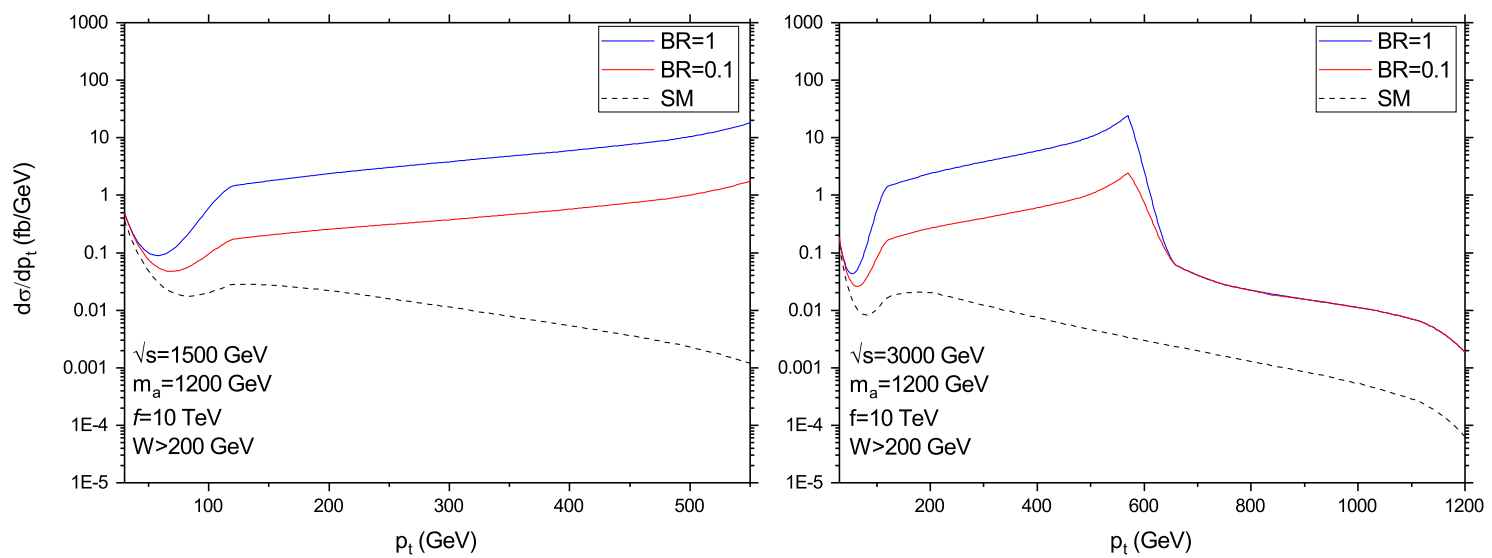

Figure 2. The differential cross sections for the process $\gamma \gamma \rightarrow \gamma \gamma$ at the CLIC for the CB initial photons with the ALP mass $m_{a}=1200 \mathrm{GeV}$, coupling constant $f=10 \mathrm{TeV}$, and cut $W>200 \mathrm{GeV}$ imposed on the diphoton invariant mass $W$. The invariant energy is equal to $\sqrt{s}=1500$ (3000) $\mathrm{GeV}$ in the left (right) panel. The curves both for $\operatorname{Br}(a \rightarrow \gamma \gamma)=1.0$ and $\operatorname{Br}(a \rightarrow \gamma \gamma)=0.1$ are shown. The dashed lines denote the SM contributions.
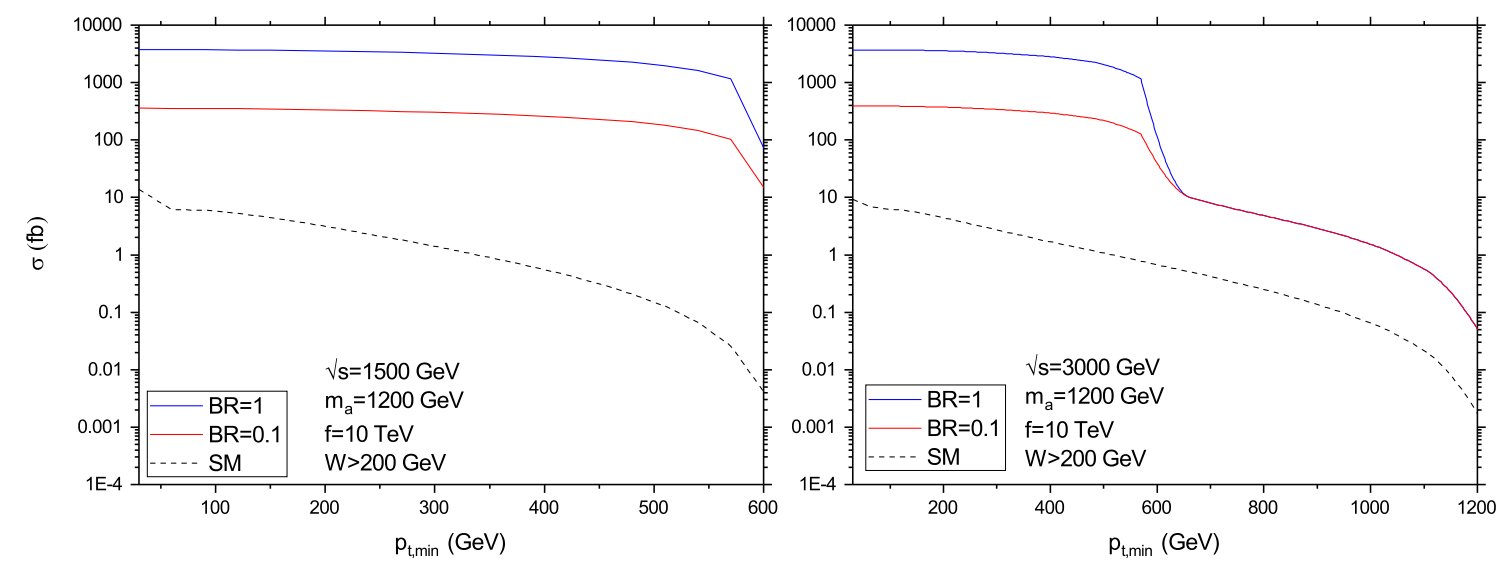

Figure 3. The same as in figure 2, but for the total cross sections as functions of the transverse momenta cutoff $p_{\mathrm{t}, \mathrm{min}}$ of the final photons.

mass $m_{a}$ and its coupling $f$ are chosen to be equal to $1200 \mathrm{GeV}$ and $10 \mathrm{TeV}$, respectively. In order to reduce the SM background, we have imposed the cut $W=m_{\gamma \gamma}>200 \mathrm{GeV}$. The curves are presented for two values of the ALP branching into two photons $\mathrm{Br}=\operatorname{Br}(a \rightarrow$ $\gamma \gamma)$. For this differential cross sections, the virtual production of the ALP dominates the SM light-by-light subprocess for $p_{t}>100 \mathrm{GeV}$ region. The total cross sections $\sigma\left(p_{t}>\right.$ $\left.p_{t, \min }\right)$ as functions of the minimal transverse momenta of the final photons $p_{t, \text { min }}$ are shown in figure 3. It can be seen from this figure that the deviation from the SM gets higher as the $p_{t}$-cut increases. Moreover, while the SM cross section decreases up to the value of $p_{t, \text { min }}=500 \mathrm{GeV}$, the total cross section remains almost unchanged.

Figure 4 demonstrates the dependence of the total cross sections on the ALP mass for two fixed values of the ALP coupling $f=10 \mathrm{TeV}$ (in the left panel) and $f=100 \mathrm{TeV}$ (in 
the right panel). Since in the mass region $m_{a}=1000-2500 \mathrm{GeV}$, the dominant dependence of the cross sections scales as $1 / f^{2}$, one can assume that it comes from the interference piece. However, it is not a case. Our calculations have shown that the pure axion matrix element $M_{a}$ in (2.3) is dominant. Moreover, $t$ - and $u$-channel terms in eqs. (2.7)-(2.9) scale as $1 / f^{4}$ and are negligible with respect to $s$-channel terms in eqs. (2.4) and (2.9). The main contribution comes from the resonance region $s \sim m_{a}^{2}$ in the $s$-channel terms of $M_{a}$. As a result, in the mass region $m_{a}=1000-2500 \mathrm{GeV}$ we get

$$
\sigma \sim \frac{1}{f^{2}} \operatorname{Br}(\mathrm{a} \rightarrow \gamma \gamma)
$$

in a qualitative agreement with figure 4. For more details, see appendix A.

One can also see that the cross sections are very sensitive to the parameter $m_{a}$ in the interval $m_{a}=1000-2500 \mathrm{GeV}$, in which it is approximately two orders of magnitude greater than for $m_{a}$ outside of this mass range. It is not surprising that this is the region where the value of the ALP coupling constant $f$ is mostly restricted by the light-by-light process, see figures 5,6 . In these figures, we have applied the cut $p_{t}>500 \mathrm{GeV}$ in order to suppress SM cross sections relative to total cross sections as we analyzed from the figure 3. In this analysis, we have used the following statistical significance $(S S)$ formula [109]

$$
S S=\sqrt{2[(S+B) \ln (1+S / B)-S]} .
$$

Here $S$ and $B$ are the numbers of the signal and background events, respectively. It can be obtained that $S S \simeq S / \sqrt{B}$ for $S \ll B$. It is assumed that the uncertainty of the background is negligible.

Our obtained exclusion regions should be compared with the current exclusion regions on the ALP coupling and ALP mass presented in figure 7, especially with that obtained for the process $p p \rightarrow p(\gamma \gamma \rightarrow \gamma \gamma) p$ at the LHC [83, 84]. This comparison demonstrates the great potential of the light-by-light scattering at the CLIC. Our 95\% C.L. parameter exclusion region is presented in figure 5 for $\sqrt{s}=1500 \mathrm{GeV}$ and $L=2500 \mathrm{fb}^{-1}$ using $\operatorname{Br}(a \rightarrow$ $\gamma \gamma)=1.0,0.5$, and 0.1 . The best bounds are achieved for $\operatorname{Br}(a \rightarrow \gamma \gamma)=1.0$. This figure shows the upper bound $f^{-1}<5.5 \times 10^{-2} \mathrm{TeV}^{-1}$ for the ALP mass interval $10 \mathrm{GeV}-800 \mathrm{GeV}$, while the light-by-light scattering at the LHC gives the bound $f^{-1}<4 \times 10^{-1} \mathrm{TeV}^{-1}$ for the same mass interval. Moreover, we have obtained the very strong upper bound on $f^{-1}$, which is of the order of $10^{-4} \mathrm{TeV}^{-1}$ for the mass range $m_{a}=1000-1200 \mathrm{GeV}$. The best limit for the $p p \rightarrow p(\gamma \gamma \rightarrow \gamma \gamma) p$ is of the order of $10^{-2} \mathrm{TeV}^{-1}$ for the mass range $m_{a}=600-800 \mathrm{GeV}$, as seen from figure 7. The $95 \%$ C.L. exclusion region for $\sqrt{s}=3000 \mathrm{GeV}$ and $L=5000 \mathrm{fb}^{-1}$ is presented in figure 6 . It demonstrates the wider exclusion regions. In particular, one can be derived the upper bound $f^{-1}<3 \times 10^{-2} \mathrm{TeV}^{-1}$ for the ALP mass interval $10 \mathrm{GeV}-$ $800 \mathrm{GeV}$. The stronger bounds on $f^{-1}$ have been obtained, which are of the order of $10^{-4} \mathrm{TeV}^{-1}$ for the mass range $m_{a}=1000-2400 \mathrm{GeV}$ and $\operatorname{Br}(a \rightarrow \gamma \gamma)=1.0$.

The sensitivity region in figure 6 is limited to a rather sharp region. It is due to the fact that the cross section is large only in the mass range $1000-2500 \mathrm{GeV}$, see figure 4 . In this region the axion term dominates, while outside it the contributions from the axion and SM terms are comparable and they partially cancel each other. By comparison the exclusion 

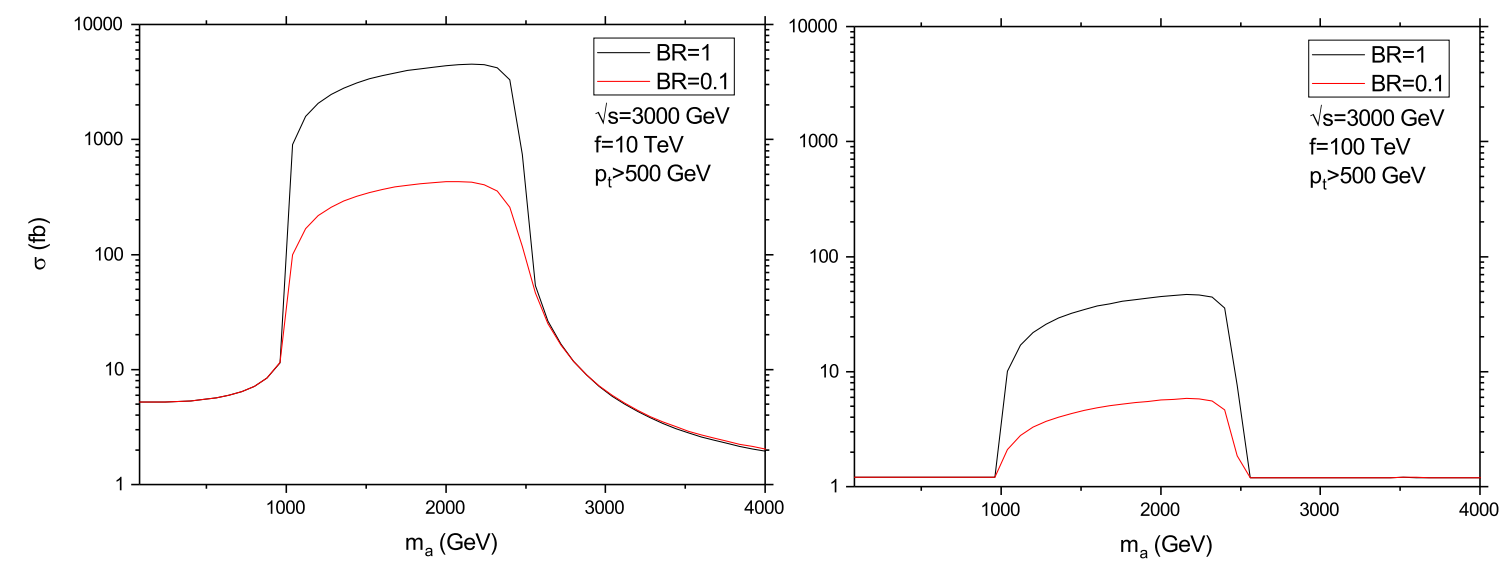

Figure 4. The total cross sections for the process $\gamma \gamma \rightarrow \gamma \gamma$ at the CLIC for the CB initial photons as functions of the ALP mass $m_{a}$ for $f=10 \mathrm{TeV}$ and $f=100 \mathrm{TeV}$ with two values of $\operatorname{Br}(a \rightarrow \gamma \gamma)$.

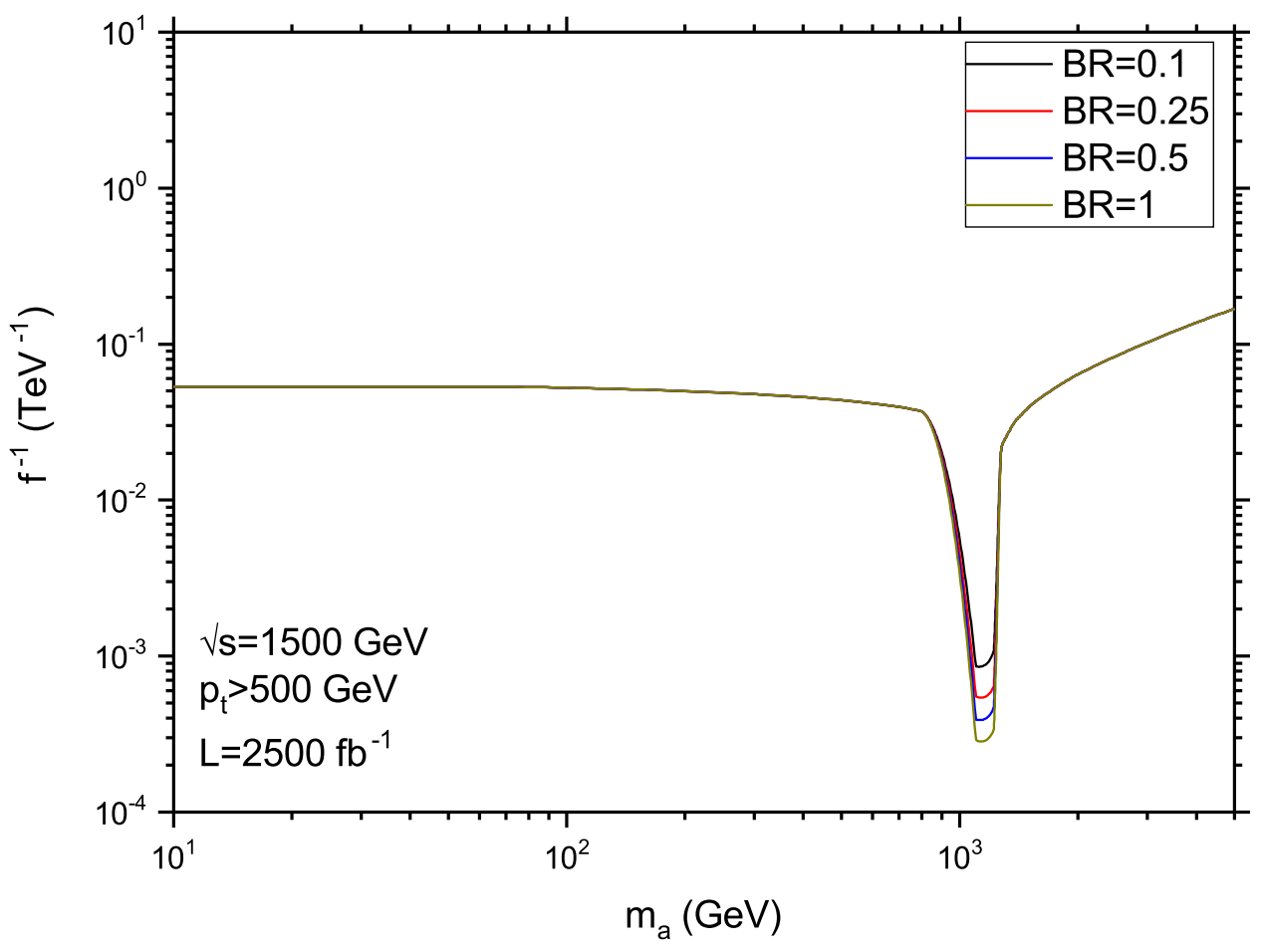

Figure 5. The 95\% C.L. CLIC exclusion region for the process $\gamma \gamma \rightarrow \gamma \gamma$ for the CB initial photons with the invariant energy $\sqrt{s}=1500 \mathrm{GeV}$, cut $W>200 \mathrm{GeV}$ on the photon invariant mass, integrated luminosity $L=2500 \mathrm{fb}^{-1}$, and different values of $\operatorname{Br}(a \rightarrow \gamma \gamma)$. 


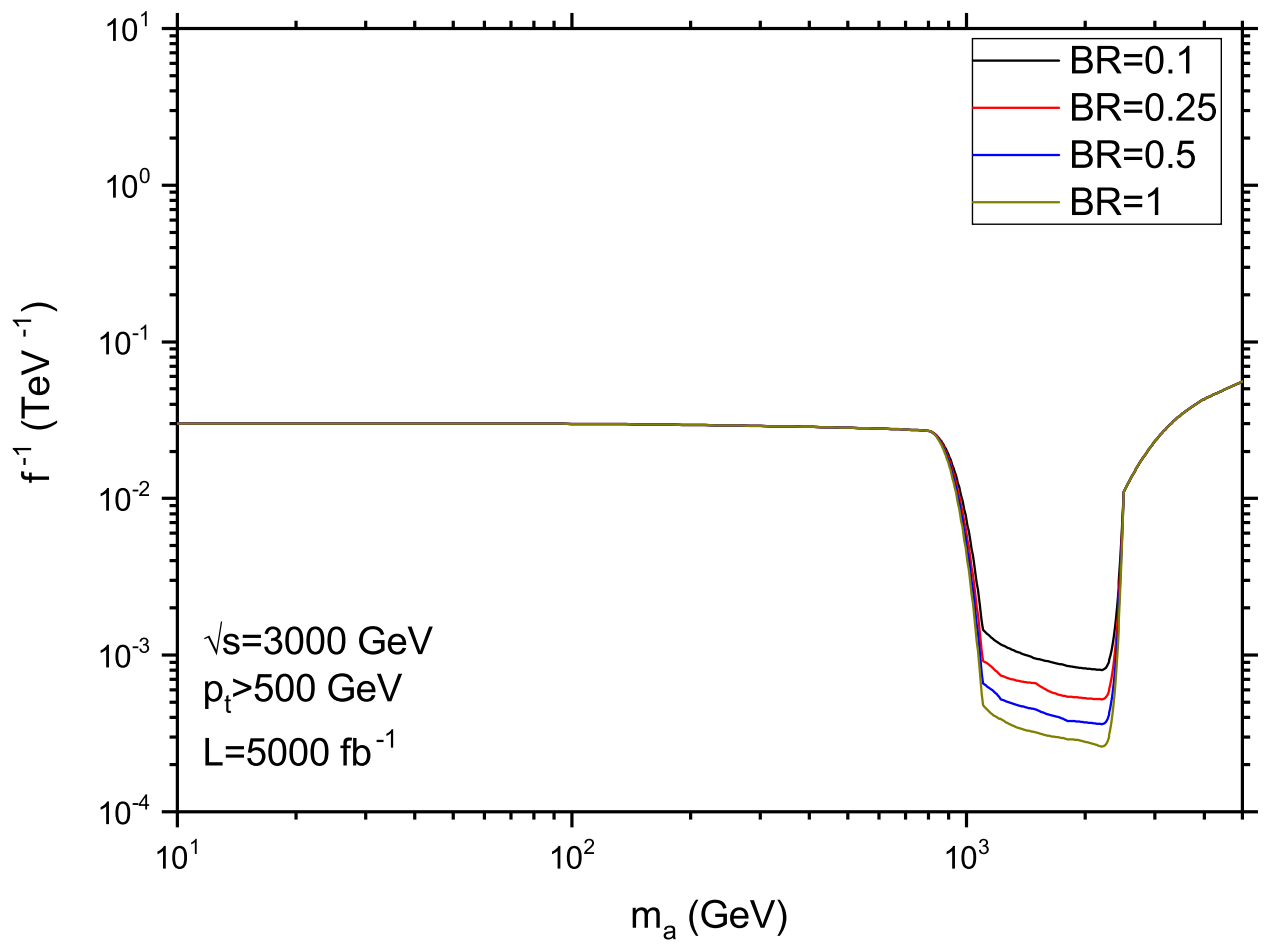

Figure 6. The as in figure 5 , but for $\sqrt{s}=3000 \mathrm{GeV}$ and $L=5000 \mathrm{fb}^{-1}$.

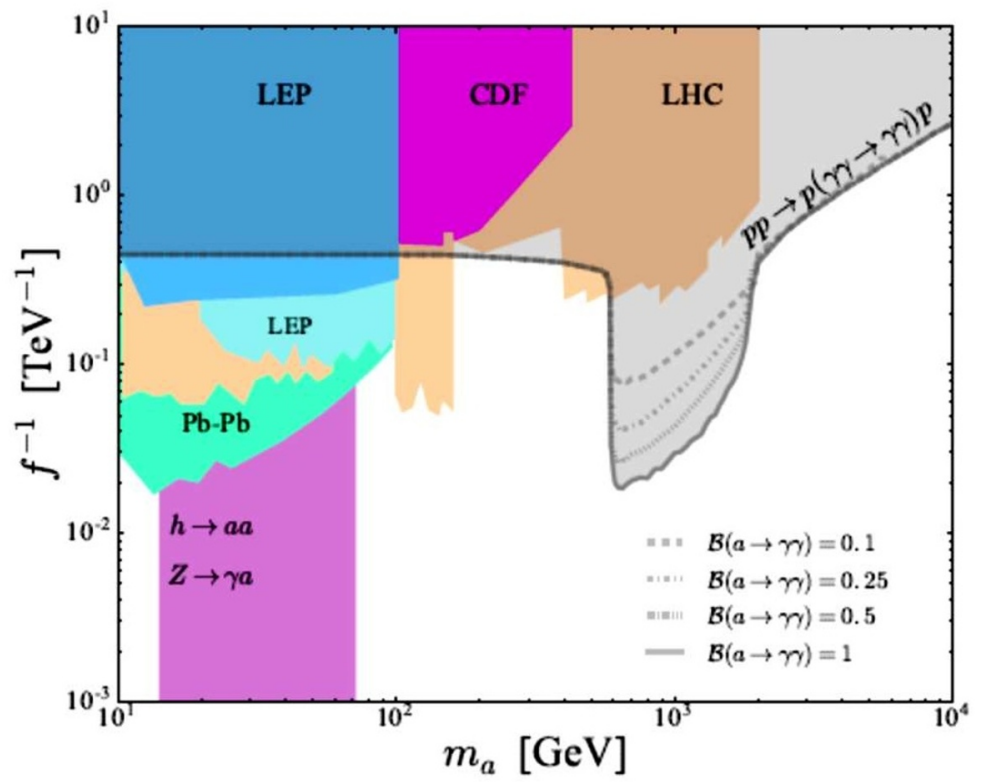

Figure 7. The $95 \%$ C.L. current exclusion regions for different values of $\operatorname{Br}(a \rightarrow \gamma \gamma)[83,84]$. 

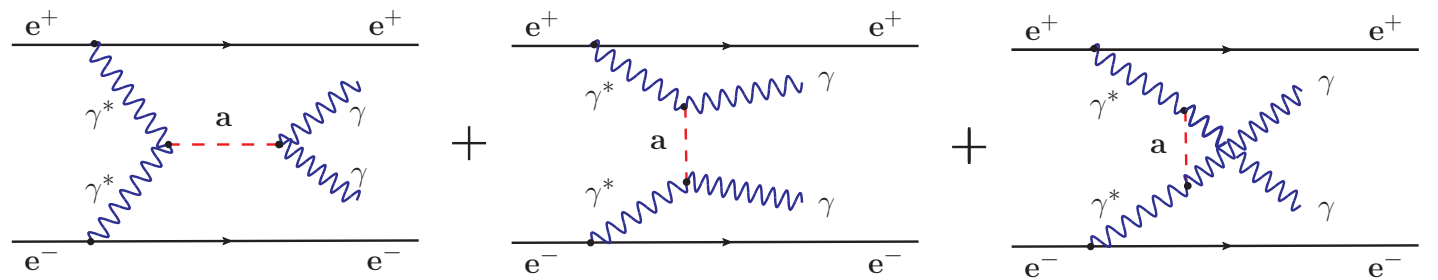

Figure 8. The Feynman diagrams describing photon-induced light-by-light virtual production of the axion-like particle $a$ in $e^{+} e^{-}$collision.

region in figure 6 with that for the process $p p \rightarrow p(\gamma \gamma \rightarrow \gamma \gamma) p$ in figure 7 , we have to conclude that the above mentioned behavior of the sensitivity is really related to a specific dependence of the subprocess $\gamma \gamma \rightarrow \gamma \gamma$ on the axion mass as discussed in appendix A.

\subsection{Weizsäcker-Williams photons}

The photon-photon interactions can be realized at the CLIC in a different way, using almost real photons emitted from incoming electron beams. These processes can be studied in the framework of the Weizsäcker-Williams approximation (WWA) [110]-[114].

In this approximation, incoming electrons scatter at very small angles. Therefore, electrons can not be caught in the main detector. If scattered electrons of the beams are observed, minimal and maximal values of scattered photon energies can be determined. Otherwise, energy or momentum cuts imposed on final state particles can be used to specify the minimum photon energy. The photon virtuality varies in the following kinematical range

$$
Q_{\min }^{2} \leq Q^{2} \leq Q_{\max }^{2}
$$

where $Q^{2}=-q^{2}$ ( $q$ is the photon momentum), and

$$
Q_{\min }^{2}=\frac{m_{e}^{2} x^{2}}{1-x}, \quad Q_{\max }^{2}=\frac{4 E_{e}^{2}}{1-x} .
$$

Here $E_{e}$ is the energy of the incoming electron, $E_{\gamma}$ is the photon energy and $x=E_{\gamma} / E_{e}$. The scattered electrons have very small scattering angles from the beam direction, and their transverse momenta are small. Therefore, due to momentum conservation, the transverse momenta of the emitted photons should also be very small. This means that the virtuality of the photon $Q^{2}$ in WWA is very small (almost real). In an experiment performed by the DELPHI Collaboration, it was observed that the virtualities of $90 \%$ photons are less than $1 \mathrm{GeV}^{2}$ using appropriate experimental techniques [115]. The WWA is also useful for experimental studies due to it allows us to find cross sections for the process $e^{-} e^{+} \rightarrow e^{-} X e^{+}$via subprocess $\gamma \gamma \rightarrow X$ [116]. In the literature, there are many papers on photon-induced processes, see, for instance, [115, 117]-[124].

In the WWA, the photons have the following spectrum

$$
f_{\gamma / e}(x)=\frac{\alpha}{\pi}\left[\left(\frac{1-x+x^{2} / 2}{x}\right) \log \frac{Q_{\max }^{2}}{Q_{\min }^{2}}-\frac{m_{e}^{2} x}{Q_{\min }^{2}}\left(1-\frac{Q_{\min }^{2}}{Q_{\max }^{2}}\right)\right] .
$$



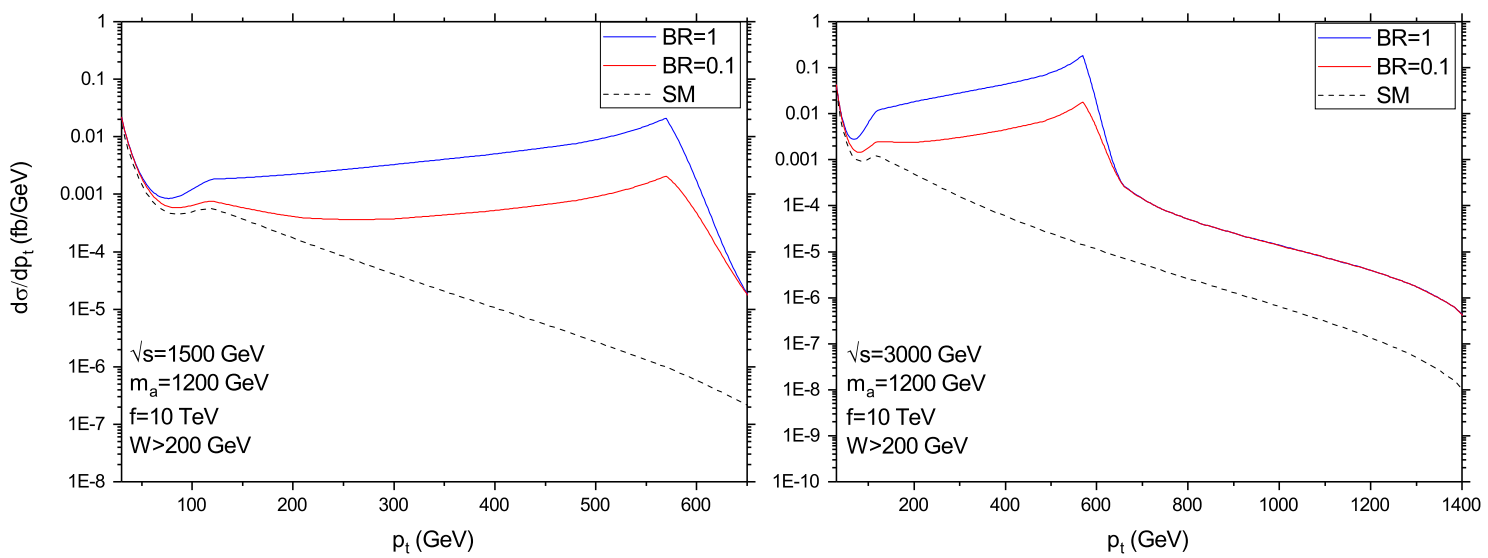

Figure 9. The differential cross sections for the process $e^{+} e^{-} \rightarrow e^{+} \gamma \gamma e^{-} \rightarrow e^{+} a e^{-} \rightarrow e^{+} \gamma \gamma e^{-}$ at the CLIC in the WWA for the initial photons in the subprocess $\gamma \gamma \rightarrow \gamma \gamma$ as functions of the transverse momenta of the final photons $p_{t}$ for the ALP mass $m_{a}=1200 \mathrm{GeV}$ and coupling constant $f=10 \mathrm{TeV}$. The invariant energy is equal to $\sqrt{s}=1500$ (3000) GeV on the left (right) panel. The curves for both $\operatorname{Br}(a \rightarrow \gamma \gamma)=1.0$ and $\operatorname{Br}(a \rightarrow \gamma \gamma)=0.1$ are shown. The dashed lines denote the SM contributions.

Here $m_{e}$ is the electron mass and $\alpha$ is the fine structure constant. The cross section of the process $e^{-} e^{+} \rightarrow e^{-} \gamma \gamma e^{+}$can be calculated using formula (2.25) with the replacement $y_{\max }=z_{\max }=1-m_{e} / E_{e}$. In the light of above arguments, in what follows, we take $Q_{\max }^{2}=2 \mathrm{GeV}^{2}$.

In addition to the backgrounds mentioned in subsection 2.1, possible backgrounds also came from $\gamma \gamma \rightarrow e^{+} e^{-} \gamma \gamma$ and $Z Z$-induced processes. The first one was estimated in [95] to be below $1 \%$. The second one may not be taken into account since the $Z Z$ luminosity is approximately 100 times smaller than the $\gamma \gamma$ luminosity [125].

The results of our calculations of the differential and total cross sections are presented in figures 9 and 10. They should be compared with the cross sections for the process induced by the CB photons shown in figures 2 and 3. The WWA cross sections have appeared to be approximately $10^{4}\left(10^{2}\right)$ times smaller that the CB cross sections for $\sqrt{s}=1500 \mathrm{GeV}$ $(\sqrt{s}=3000 \mathrm{GeV})$.

The same one can see in figure 11, where the total cross section for the process $e^{+} e^{-} \rightarrow$ $e^{+} \gamma \gamma e^{-} \rightarrow e^{+} a e^{-} \rightarrow e^{+} \gamma \gamma e^{-}$is shown as a function of the ALP mass $m_{a}$. For the ALP branching ratio $\operatorname{Br}(a \rightarrow \gamma \gamma)=1.0$, there are big bumps in the curves in the mass region $1000 \mathrm{GeV}-3000 \mathrm{GeV}$ for both values of the collision energy $\sqrt{s}$.

Figure 12 gives the $95 \%$ C.L. CLIC exclusion region in the $\left(m_{a}, f^{-1}\right)$ plane in the case when the subprocess $\gamma \gamma \rightarrow \gamma \gamma$ is induced by the WWA photons with $\sqrt{s}=1500 \mathrm{GeV}$ and $L=2500 \mathrm{fb}^{-1}$. As one can see from this figure, the bounds are of the order of $10^{-1} \mathrm{TeV}^{-1}$ in the mass regions $10 \mathrm{GeV}-1000 \mathrm{GeV}$. In the narrow mass region $1000 \mathrm{GeV}-1500 \mathrm{GeV}$ it is obtained to be of the order of $10^{-3} \mathrm{TeV}^{-1}$. Similarly, figure 13 shows the $95 \%$ C.L. exclusion region in the $\left(m_{a}, f^{-1}\right)$ plane for $\sqrt{s}=3000 \mathrm{GeV}$ and $L=5000 \mathrm{fb}^{-1}$. In the mass region $10 \mathrm{GeV}-1000 \mathrm{GeV}$ the bounds on $f^{-1}$ are of the order of $10^{-1} \mathrm{TeV}^{-1}$. In the mass 

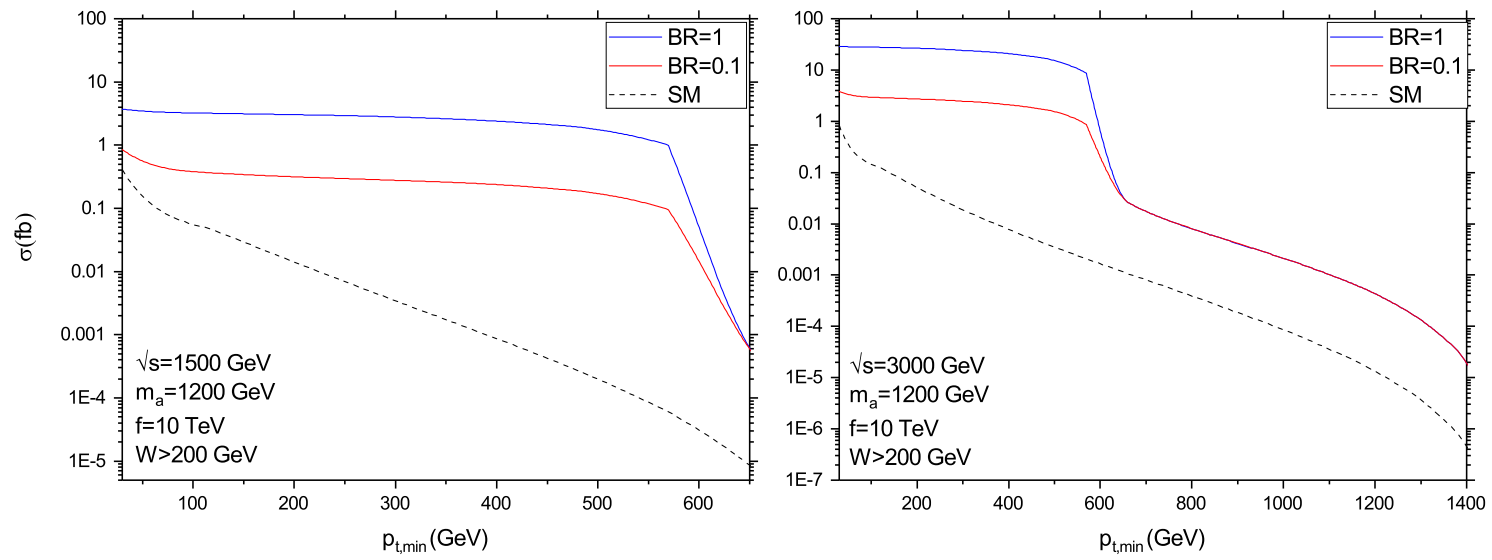

Figure 10. The same as in figure 9, but for the total cross sections as functions of the transverse momenta cutoff $p_{\mathrm{t}, \mathrm{min}}$ of the final photons.
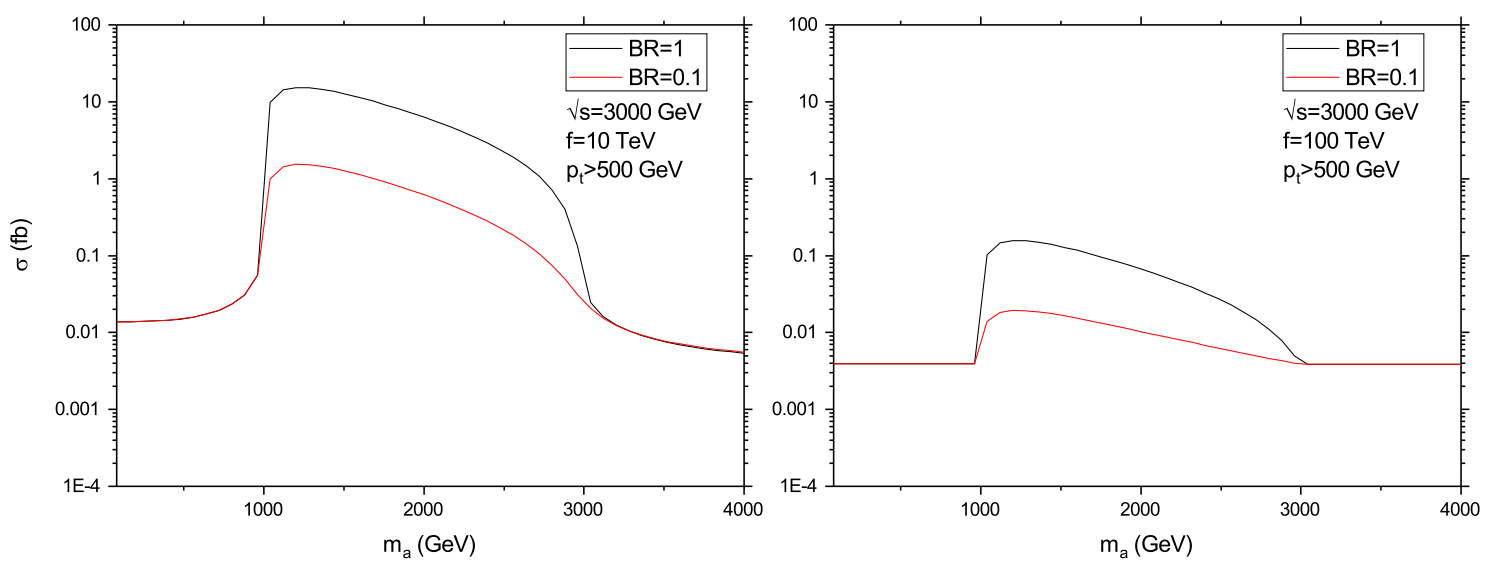

Figure 11. The total cross sections for the process $e^{+} e^{-} \rightarrow e^{+} \gamma \gamma e^{-} \rightarrow e^{+} a e^{-} \rightarrow e^{+} \gamma \gamma e^{-}$at the CLIC in the WWA for the initial photons as functions of the ALP mass $m_{a}$ for $f=10 \mathrm{TeV}$ and $f=100 \mathrm{TeV}$ with two values of $\operatorname{Br}(a \rightarrow \gamma \gamma)$.

range $1000 \mathrm{GeV}-1500 \mathrm{GeV}$, these bounds reach the value $1 \times 10^{-3} \mathrm{TeV}^{-1}$. For both $\sqrt{s}$, the bounds are much weaker than those for the CB initial photons.

\section{Conclusions}

We have studied the possibility to search for heavy axion-like particles in the process $\gamma \gamma \rightarrow \gamma \gamma$ with Compton backscattered initial photons and process $e^{+} e^{-} \rightarrow e^{+} e^{-} \gamma \gamma$ induced by light-by-light scattering with Weizsäcker-Williams initial photons at the CLIC. The calculations were made for the collision energy $\sqrt{s}=1500 \mathrm{GeV}$ (2nd stage of the CLIC) and integrated luminosity $L=2500 \mathrm{fb}^{-1}$, as well as for the energy $\sqrt{s}=3000 \mathrm{GeV}$ and integrated luminosity $L=5000 \mathrm{fb}^{-1}$ (3rd stage of the CLIC). It was assumed that the pseudoscalar ALP interacts with photons via CP-even term in the Lagrangian (2.1). 


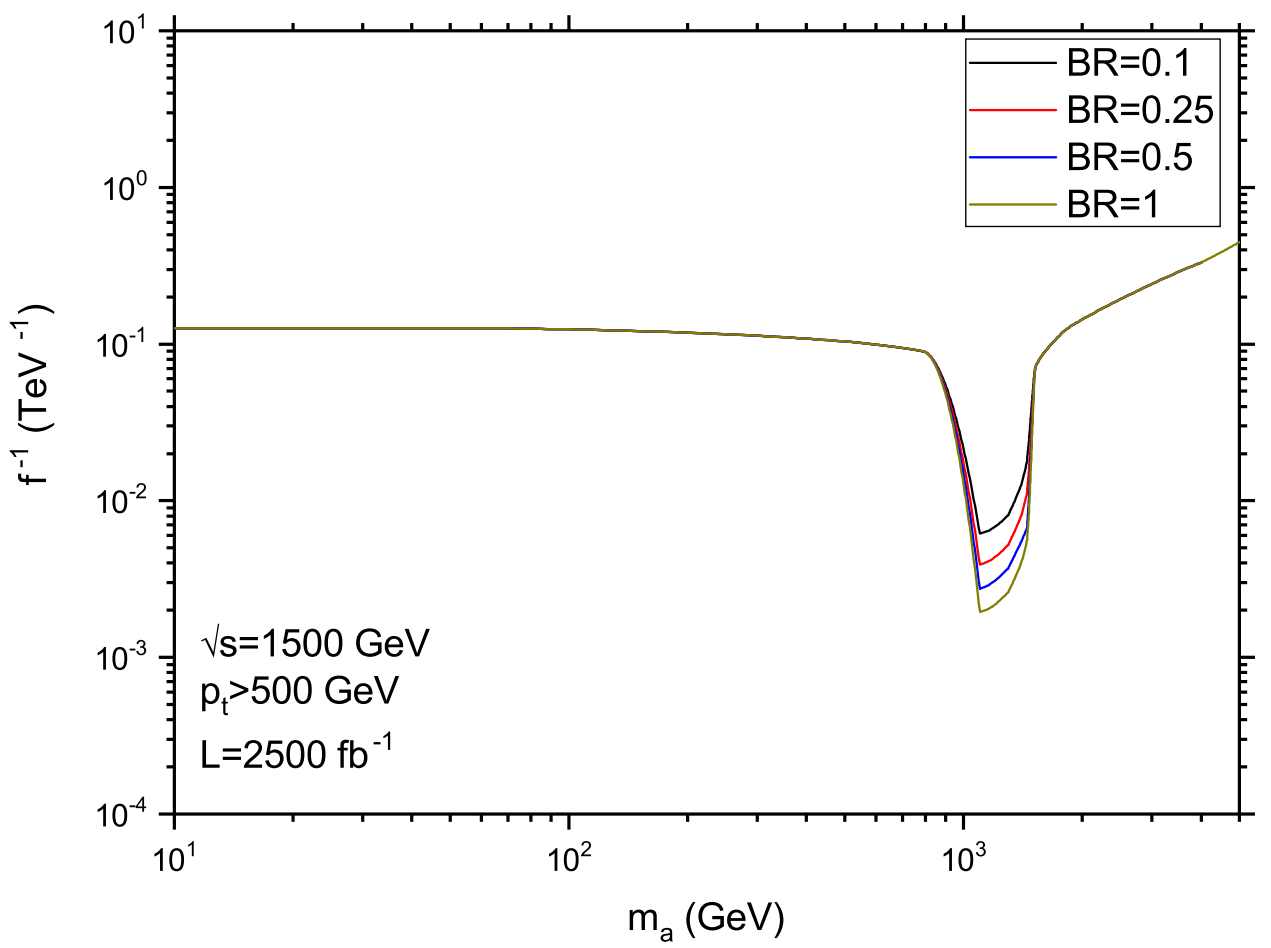

Figure 12. The $95 \%$ C.L. CLIC exclusion region for the process $e^{+} e^{-} \rightarrow e^{+} \gamma \gamma e^{-} \rightarrow e^{+} a e^{-} \rightarrow$ $e^{+} \gamma \gamma e^{-}$with the invariant energy $\sqrt{s}=1500 \mathrm{GeV}$, transverse momentum cut on the final photons $p_{t}=500 \mathrm{GeV}$, integrated luminosity $L=2500 \mathrm{fb}^{-1}$, and different values of $\operatorname{Br}(a \rightarrow \gamma \gamma)$. The WWA for the initial photons in the subprocess $\gamma \gamma \rightarrow \gamma \gamma$ is used.

We $95 \%$ C.L. exclusion regions in the plane $\left(m_{a}, f^{-1}\right)$, where $m_{a}$ is the ALP mass, $f^{-1}$ ALP-photon coupling, are given. The results are presented for two values of $\sqrt{s}$ and $L$ as functions of the ALP branching ratio into photons $\operatorname{Br}(a \rightarrow \gamma \gamma)$. The best bounds are obtained for $\operatorname{Br}(a \rightarrow \gamma \gamma)=1$. Our calculations have shown that the numerical results remain almost the same if we take into account the $\mathrm{CP}$-odd term instead of the $\mathrm{CP}$-even one in the Lagrangian (2.1), with the same coupling $f^{-1}$.

By comparing our exclusion regions with other collider exclusion regions, we may conclude that the ALP search at the CLIC has the great physics potential of searching for the ALPs, especially, in the mass region $1 \mathrm{TeV}-2.4 \mathrm{TeV}$, for the collision energy $\sqrt{s}=$ $3000 \mathrm{GeV}$ and integrated luminosity $L=5000 \mathrm{fb}^{-1}$. In particular, our bounds are much stronger than recently obtained bounds for the ALP virtual production in the process $p(\gamma \gamma \rightarrow \gamma \gamma) p$ at the LHC $[83,84]$.

\section{A Dependence of total cross section on parameters of axion-like particle}

Here we obtain an approximate formula for the cross section with the CB initial photons. As it was already mentioned in the text, in the mass region $1000-2500 \mathrm{GeV}$ the dominant contribution to the cross section comes from the $s$-channel terms in $M_{a}$. Let us put $M=$ 


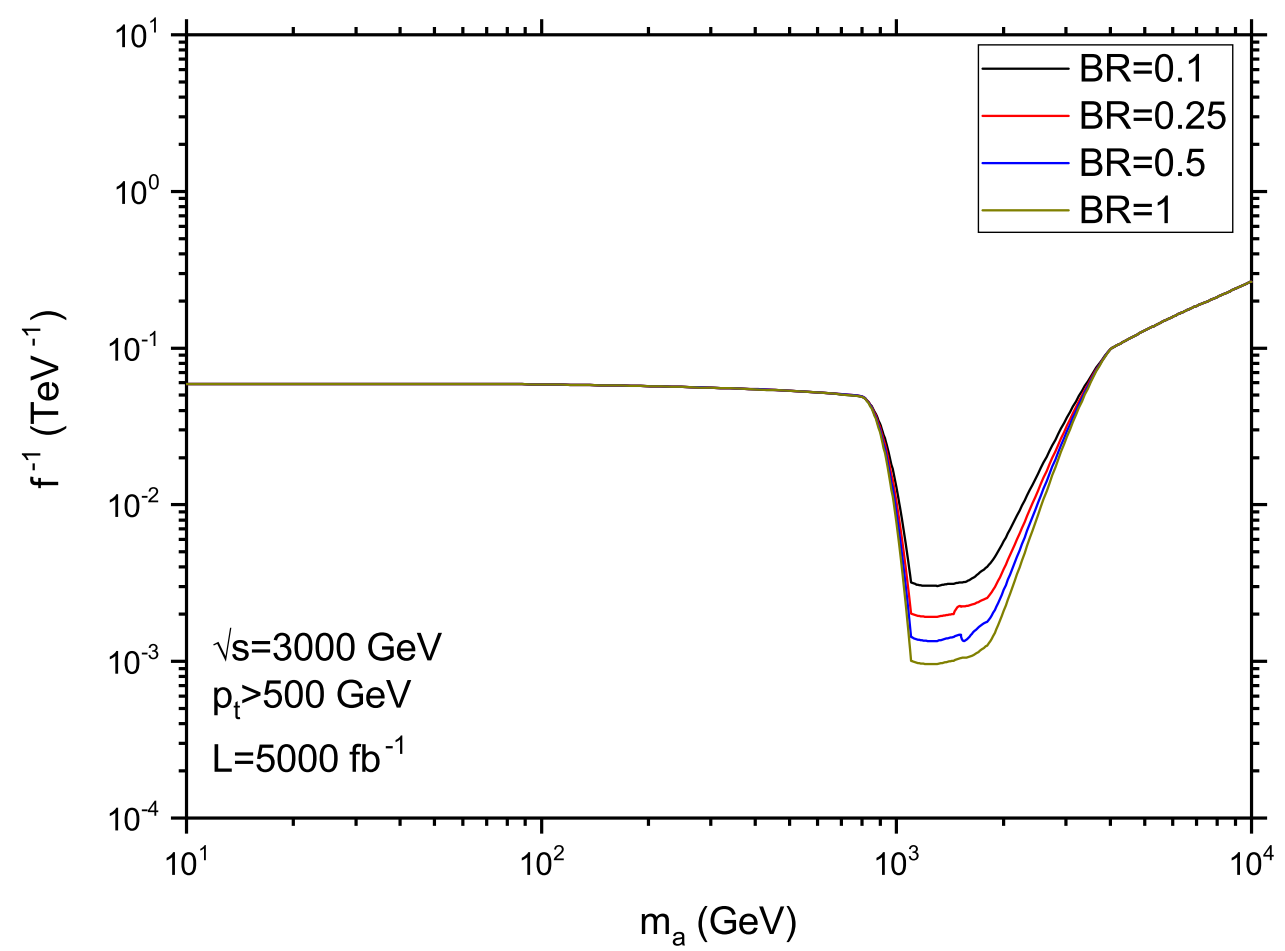

Figure 13. The same as in figure 12 , but for $\sqrt{s}=3000 \mathrm{GeV}$ and $L=5000 \mathrm{fb}^{-1}$.

$M_{++++}(2.4)$

$$
M=-\frac{4}{f^{2}} \frac{s^{2}}{s-m_{a}^{2}+i m_{a} \Gamma_{a}} .
$$

Then

$$
|M|^{2}=\frac{16}{f^{4}} \frac{s^{4}}{\left(s-m_{a}^{2}\right)^{2}+m_{a}^{2} \Gamma_{a}^{2}} .
$$

We get from (A.2) that at high energy

$$
\left.|M|^{2}\right|_{s \gg m_{a}^{2}} \sim \frac{s^{2}}{f^{4}} .
$$

However, simple dimensional arguments are not valid in the most important resonance region $s \sim m_{a}^{2}$ in which

$$
\left.|M|^{2}\right|_{s \sim m_{a}^{2}} \sim \frac{m_{a}^{6}}{f^{4} \Gamma_{a}^{2}} .
$$

Since $M$ depends only on $s$, we find from the relation

$$
\frac{d \hat{\sigma}(s)}{d \Omega}=\frac{|M|^{2}}{64 \pi^{2} s}
$$

that the cross section of the subprocess $\gamma \gamma \rightarrow \gamma \gamma$ is equal to

$$
\hat{\sigma}(s)=\frac{1}{16 \pi s}|M|^{2} .
$$


The integrations in eq. (2.25) can be rewritten as follows

$$
2 z d z \frac{d y}{y}=d x_{1} d x_{2} \sim \frac{d x_{1}}{x_{1}} \frac{d s}{4 E_{e}^{2}} .
$$

Then the cross section of the process under consideration is given by the integral

$$
\sigma=\frac{1}{4 E_{e}^{2}} \int d s \frac{1}{16 \pi s}|M|^{2} .
$$

Let us estimate the contribution to $\sigma$ from the resonance region

$$
m_{a}^{2}-C m_{a} \Gamma_{a} \leqslant s \leqslant m_{a}^{2}+C m_{a} \Gamma_{a}
$$

where $C$ is a constant of order $\mathrm{O}(1)$. After introducing variable $x=\left(s-m_{a}^{2}\right) /\left(m_{a} \Gamma_{a}\right)$, we get

$$
\sigma=\frac{1}{f^{2}} \frac{m_{a}^{2}}{E_{e}^{2}} \operatorname{Br}(a \rightarrow \gamma \gamma) \int_{-C}^{C} d x \frac{1}{x^{2}+1} .
$$

In order to numerically evaluate the cross section, we put $C=1$. Then we obtain the estimate

$$
\sigma \simeq 0.39 \times 10^{6} \frac{\pi}{2}\left(\frac{\mathrm{TeV}}{f}\right)^{2}\left(\frac{m_{a}}{E_{e}}\right)^{2} \operatorname{Br}(a \rightarrow \gamma \gamma) \mathrm{fb} .
$$

Comparing this formula with figure 4 , we see that it gives a correct dependence of the cross section on the parameters $f, m_{a}$ and $\operatorname{Br}(a \rightarrow \gamma \gamma$ in the mass region $1100-2500 \mathrm{GeV}$. As for numerical values of $\sigma$, we find, for example, for $E_{e}=3.0 \mathrm{TeV}, f=10 \mathrm{TeV}, m_{a}=1.5 \mathrm{TeV}$, and $\operatorname{Br}(a \rightarrow \gamma \gamma)=1.0$

$$
\sigma \simeq 1.6 \times 10^{3} \mathrm{fb}
$$

An accurate integration with an account of the photon spectra should modify the numerical factor in the right-hand-side of eq. (A.11). This formula must be regarded only as an illustration of how the main contribution to the cross section comes from the resonance region of the pure axion amplitude.

Open Access. This article is distributed under the terms of the Creative Commons Attribution License (CC-BY 4.0), which permits any use, distribution and reproduction in any medium, provided the original author(s) and source are credited.

\section{References}

[1] S. Weinberg, The U(1) problem, Phys. Rev. D 11 (1975) 3583 [inSPIRE].

[2] S.L. Adler, Axial vector vertex in spinor electrodynamics, Phys. Rev. 177 (1969) 2426 [INSPIRE].

[3] J.S. Bell and R. Jackiw, A PCAC puzzle: $\pi^{0} \rightarrow \gamma \gamma$ in the $\sigma$ model, Nuovo Cim. A 60 (1969) 47 [INSPIRE].

[4] G. 't Hooft, Symmetry breaking through Bell-Jackiw anomalies, Phys. Rev. Lett. 37 (1976) 8 [inSPIRE]. 
[5] G. 't Hooft, Computation of the quantum effects due to a four-dimensional pseudoparticle, Phys. Rev. D 14 (1976) 3432 [Erratum ibid. 18 (1978) 2199] [InSPIRE].

[6] A.A. Belavin, A.M. Polyakov, A.S. Schwartz and Y. Tyupkin, Pseudoparticle solutions of the Yang-Mills equations, Phys. Lett. B 59 (1975) 85 [inSPIRE].

[7] Particle Data Group collaboration, Review of particle physics, Phys. Rev. D 98 (2018) 030001 [inSPIRE].

[8] R.D. Peccei and H.R. Quinn, CP conservation in the presence of instantons, Phys. Rev. Lett. 38 (1977) 1440 [INSPIRE].

[9] S. Weinberg, A new light boson?, Phys. Rev. Lett. 40 (1978) 223 [INSPIRE].

[10] F. Wilczek, Problem of strong $P$ and $T$ invariance in the presence of instantons, Phys. Rev. Lett. 40 (1978) 279 [INSPIRE].

[11] J.E. Kim, Weak interaction singlet and strong CP invariance, Phys. Rev. Lett. 43 (1979) 103 [INSPIRE].

[12] M.A. Shifman, A.I. Vainshtein and V.I. Zakharov, Can confinement ensure natural CP invariance of strong interactions?, Nucl. Phys. B 166 (1980) 493 [INSPIRE].

[13] M. Dine, W. Fischler and M. Srednicki, A simple solution to the strong CP problem with a harmless axion, Phys. Lett. B 104 (1981) 199 [INSPIRE].

[14] A.R. Zhitnitsky, On possible suppression of the axion hadron interactions, Sov. J. Nucl. Phys. 31 (1980) 260 [Yad. Fiz. 31 (1980) 497] [INSPIRE].

[15] P. Svrček and E. Witten, Axions in string theory, JHEP 06 (2006) 051 [hep-th/0605206] [INSPIRE].

[16] J.P. Conlon, The QCD axion and moduli stabilisation, JHEP 05 (2006) 078 [hep-th/0602233] [INSPIRE].

[17] M. Cicoli, M. Goodsell and A. Ringwald, The type IIB string axiverse and its low-energy phenomenology, JHEP 10 (2012) 146 [arXiv:1206.0819] [INSPIRE].

[18] J. Halverson, C. Long, B. Nelson and G. Salinas, Towards string theory expectations for photon couplings to axionlike particles, Phys. Rev. D 100 (2019) 106010 [arXiv: 1909.05257] [INSPIRE].

[19] E. Massó and R. Toldrà, On a light spinless particle coupled to photons, Phys. Rev. D 52 (1995) 1755 [hep-ph/9503293] [INSPIRE].

[20] B. Bellazzini, A. Mariotti, D. Redigolo, F. Sala and J. Serra, R-axion at colliders, Phys. Rev. Lett. 119 (2017) 141804 [arXiv:1702.02152] [INSPIRE].

[21] V.A. Rubakov, Grand unification and heavy axion, JETP Lett. 65 (1997) 621 [Pisma Zh. Exsp. Teor. Fiz. 65 (1997) 590] [hep-ph/9703409] [INSPIRE].

[22] G. Grilli di Cortona, E. Hardy, J. Pardo Vega and G. Villadoro, The QCD axion, precisely, JHEP 01 (2016) 034 [arXiv: 1511.02867] [INSPIRE].

[23] R.T. Co, L.J. Hall and K. Harigaya, Kinetic misalignment mechanism, arXiv:1910.14152 [INSPIRE].

[24] C.-F. Chang and Y. Cui, New perspectives on axion misalignment mechanism, arXiv:1911.11885 [INSPIRE]. 
[25] P. Fileviez Pérez, C. Murgui and A.D. Plascencia, The QCD axion and unification, JHEP 11 (2019) 093 [arXiv: 1908.01772] [INSPIRE].

[26] C. Xiong, Axionic excitation from QCD condensate, arXiv:1912.13489 [INSPIRE].

[27] Y. Gao, T. Li and Q. Yang, The minimal UV-induced effective QCD axion theory, arXiv: 1912.12963 [INSPIRE].

[28] M.M. Anber and E. Poppitz, Deconfinement on axion domain walls, JHEP 03 (2020) 124 [arXiv: 2001.03631] [INSPIRE].

[29] H. Matsui, F. Takahashi and W. Yin, QCD axion window and false vacuum Higgs inflation, JHEP 05 (2020) 154 [arXiv: 2001. 04464] [INSPIRE].

[30] T. Gherghetta, V.V. Khoze, A. Pomarol and Y. Shirman, The axion mass from $5 D$ small instantons, JHEP 03 (2020) 063 [arXiv: 2001.05610] [INSPIRE].

[31] J.E. Kim, Light pseudoscalars, particle physics and cosmology, Phys. Rept. 150 (1987) 1 [INSPIRE].

[32] R.D. Peccei, The strong CP problem and axions, hep-ph/0607268 [INSPIRE].

[33] G.G. Raffelt, Astrophysical axion bounds, hep-ph/0611350 [INSPIRE].

[34] D.J.E. Marsh, Axions and ALPs: a very short introduction, in $13^{\text {th }}$ Patras Workshop on Axions, WIMPs and WISPs, DESY-PROC-2017-02, (2018), pg. 59 [arXiv:1712.03018] [INSPIRE].

[35] S. Chang, S. Tazawa and M. Yamaguchi, Axion model in extra dimensions with TeV scale gravity, Phys. Rev. D 61 (2000) 084005 [hep-ph/9908515] [INSPIRE].

[36] K.R. Dienes, E. Dudas and T. Gherghetta, Invisible axions and large radius compactifications, Phys. Rev. D 62 (2000) 105023 [hep-ph/9912455] [INSPIRE].

[37] E. Ma, M. Raidal and U. Sarkar, Low scale axion from large extra dimensions, Phys. Lett. B 504 (2001) 296 [hep-ph/0007321] [INSPIRE].

[38] B. Lakić, R. Horvat and M. Krčmar, Axions and large extra dimensions, Lect. Notes Phys. 741 (2008) 73 [INSPIRE].

[39] H. Collins and R. Holman, The invisible axion in a Randall-Sundrum universe, Phys. Rev. D 67 (2003) 105004 [hep-ph/0210110] [INSPIRE].

[40] T. Flacke and D. Maybury, Aspects of axion phenomenology in a slice of AdS $S_{5}$, JHEP 03 (2007) 007 [hep-ph/0612126] [INSPIRE].

[41] Y. Burnier and F. Kühnel, Axion arising from warped extra-dimensional gauge fields, Phys. Rev. D 83 (2011) 115002 [arXiv:1101.5156] [INSPIRE].

[42] J. Preskill, M.B. Wise and F. Wilczek, Cosmology of the invisible axion, Phys. Lett. B 120 (1983) 127 [inSPIRE].

[43] L.F. Abbott and P. Sikivie, A cosmological bound on the invisible axion, Phys. Lett. B 120 (1983) 133 [INSPIRE].

[44] M. Dine and W. Fischler, The not so harmless axion, Phys. Lett. B 120 (1983) 137 [INSPIRE].

[45] P. Sikivie, Axion cosmology, Lect. Notes Phys. 741 (2008) 19 [astro-ph/0610440] [INSPIRE]. 
[46] L.D. Duffy and K. van Bibber, Axions as dark matter particles, New J. Phys. 11 (2009) 105008 [arXiv:0904.3346] [InSPIRE].

[47] G. Alonso-Álvarez, R.S. Gupta, J. Jaeckel and M. Spannowsky, On the wondrous stability of ALP dark matter, JCAP 03 (2020) 052 [arXiv: 1911.07885] [INSPIRE].

[48] N. Blinov, M.J. Dolan and P. Draper, Imprints of the early universe on axion dark matter substructure, Phys. Rev. D 101 (2020) 035002 [arXiv:1911.07853] [INSPIRE].

[49] R. Lasenby, Parametrics of electromagnetic searches for axion dark matter, arXiv: 1912.11467 [INSPIRE].

[50] A. Berlin et al., Axion dark matter detection by superconducting resonant frequency conversion, arXiv: 1912.11048 [INSPIRE].

[51] Q. Yang, Probe dark matter axions using the hyperfine structure splitting of hydrogen atoms, arXiv:1912.11472 [INSPIRE].

[52] S. Basilakos, N.E. Mavromatos and J. Solà Peracaula, Quantum anomalies in string-inspired running vacuum universe: inflation and axion dark matter, Phys. Lett. B 803 (2020) 135342 [arXiv:2001.03465] [INSPIRE].

[53] D.J.E. Marsh, Axion cosmology, Phys. Rept. 643 (2016) 1 [arXiv:1510.07633] [InSPIRE].

[54] P. Di Vecchia, M. Giannotti, M. Lattanzi and A. Lindner, Round table on axions and axion-like particles, PoS (Confinement2018) 034 (2019) [arXiv: 1902.06567] [INSPIRE].

[55] N. Fonseca, E. Morgante, R. Sato and G. Servant, Axion fragmentation, JHEP 04 (2020) 010 [arXiv: 1911.08472] [INSPIRE].

[56] Z.-Q. Xia, Y.-F. Liang, L. Feng, Q. Yuan, Y.-Z. Fan and J. Wu, Searching for the possible signal of the photon-axionlike particle oscillation in the combined GeV and TeV spectra of supernova remnants, Phys. Rev. D 100 (2019) 123004 [arXiv:1911.08096] [INSPIRE].

[57] C.S. Machado, W. Ratzinger, P. Schwaller and B.A. Stefanek, Gravitational wave probes of axion-like particles, arXiv:1912.01007 [INSPIRE].

[58] L. Di Luzio, F. Mescia, E. Nardi, P. Panci and R. Ziegler, Astrophobic axions, Phys. Rev. Lett. 120 (2018) 261803 [arXiv:1712.04940] [INSPIRE].

[59] ADMX collaboration, A search for invisible axion dark matter with the axion dark matter experiment, Phys. Rev. Lett. 120 (2018) 151301 [arXiv:1804.05750] [INSPIRE].

[60] ADMX collaboration, Extended search for the invisible axion with the axion dark matter experiment, Phys. Rev. Lett. 124 (2020) 101303 [arXiv:1910.08638] [InSPIRE].

[61] HAYSTAC collaboration, Results from phase 1 of the HAYSTAC microwave cavity axion experiment, Phys. Rev. D 97 (2018) 092001 [arXiv: 1803.03690] [INSPIRE].

[62] B.T. McAllister et al., The ORGAN experiment: an axion haloscope above $15 \mathrm{GHz}$, Phys. Dark Univ. 18 (2017) 67 [arXiv:1706. 00209] [INSPIRE].

[63] Y.K. Semertzidis et al., Axion dark matter research with IBS/CAPP, arXiv:1910.11591 [INSPIRE].

[64] C.A. Thomson, B.T. McAllister, M. Goryachev, E.N. Ivanov and M.E. Tobar, Results from UPLOAD-DOWNLOAD: a phase-interferometric axion dark matter search, arXiv: 1912.07751 [INSPIRE]. 
[65] SuPERCDMS collaboration, Constraints on dark photons and axionlike particles from the SuperCDMS Soudan experiment, Phys. Rev. D 101 (2020) 052008 [arXiv:1911.11905] [INSPIRE].

[66] S. Lee, S. Ahn, J. Choi, B.R. Ko and Y.K. Semertzidis, Axion dark matter search around $6.7 \mu \mathrm{eV}$, Phys. Rev. Lett. 124 (2020) 101802 [arXiv:2001.05102] [INSPIRE].

[67] N. Crescini et al., Axion search with a quantum-limited ferromagnetic haloscope, Phys. Rev. Lett. 124 (2020) 171801 [arXiv: 2001.08940] [INSPIRE].

[68] P.W. Graham, I.G. Irastorza, S.K. Lamoreaux, A. Lindner and K.A. van Bibber, Experimental searches for the axion and axion-like particles, Ann. Rev. Nucl. Part. Sci. 65 (2015) 485 [arXiv:1602.00039] [InSPIRE].

[69] OSQAR collaboration, New exclusion limits on scalar and pseudoscalar axionlike particles from light shining through a wall, Phys. Rev. D 92 (2015) 092002 [arXiv:1506.08082] [INSPIRE].

[70] J. Jaeckel, E. Masso, J. Redondo, A. Ringwald and F. Takahashi, The need for purely laboratory-based axion-like particle searches, Phys. Rev. D 75 (2007) 013004 [hep-ph/0610203] [INSPIRE].

[71] F. Della Valle et al., The PVLAS experiment: measuring vacuum magnetic birefringence and dichroism with a birefringent Fabry-Perot cavity, Eur. Phys. J. C 76 (2016) 24 [arXiv:1510.08052] [INSPIRE].

[72] K.A. Beyer, G. Marocco, R. Bingham and G. Gregori, Axion detection through resonant photon-photon collisions, Phys. Rev. D 101 (2020) 095018 [arXiv:2001.03392] [InSPIRE].

[73] CAST collaboration, New CAST limit on the axion-photon interaction, Nature Phys. 13 (2017) 584 [arXiv:1705. 02290] [INSPIRE].

[74] A. Mariotti, D. Redigolo, F. Sala and K. Tobioka, New LHC bound on low-mass diphoton resonances, Phys. Lett. B $\mathbf{7 8 3}$ (2018) 13 [arXiv:1710.01743] [InSPIRE].

[75] M.B. Gavela, R. Houtz, P. Quilez, R. Del Rey and O. Sumensari, Flavor constraints on electroweak ALP couplings, Eur. Phys. J. C 79 (2019) 369 [arXiv:1901.02031] [InSPIRE].

[76] L. Merlo, F. Pobbe, S. Rigolin and O. Sumensari, Revisiting the production of ALPs at B-factories, JHEP 06 (2019) 091 [arXiv: 1905.03259] [INSPIRE].

[77] W.J. Marciano, A. Masiero, P. Paradisi and M. Passera, Contributions of axionlike particles to lepton dipole moments, Phys. Rev. D 94 (2016) 115033 [arXiv:1607.01022] [INSPIRE].

[78] J. Beacham et al., Physics beyond colliders at CERN: beyond the Standard Model working group report, J. Phys. G 47 (2020) 010501 [arXiv: 1901.09966] [INSPIRE].

[79] G. Alonso-Álvarez, M.B. Gavela and P. Quilez, Axion couplings to electroweak gauge bosons, Eur. Phys. J. C 79 (2019) 223 [arXiv:1811.05466] [InSPIRE].

[80] M. Bauer, M. Neubert and A. Thamm, Collider probes of axion-like particles, JHEP 12 (2017) 044 [arXiv:1708.00443] [INSPIRE].

[81] S. Knapen, T. Lin, H.K. Lou and T. Melia, Searching for axionlike particles with ultraperipheral heavy-ion collisions, Phys. Rev. Lett. 118 (2017) 171801 [arXiv: 1607.06083] [INSPIRE].

[82] S. Knapen, T. Lin, H.K. Lou and T. Melia, LHC limits on axion-like particles from heavy-ion collisions, CERN Proc. 1 (2018) 65 [arXiv: 1709.07110] [INSPIRE]. 
[83] C. Baldenegro, S. Fichet, G. von Gersdorff and C. Royon, Searching for axion-like particles with proton tagging at the LHC, JHEP 06 (2018) 131 [arXiv:1803.10835] [INSPIRE].

[84] C. Baldenegro, S. Fichet, G. von Gersdorff and C. Royon, Probing the anomalous $\gamma \gamma \gamma Z$ coupling at the LHC with proton tagging, JHEP 06 (2017) 142 [arXiv:1703.10600] [INSPIRE].

[85] C. Baldenegro, S. Hassani, C. Royon and L. Schoeffel, Extending the constraint for axion-like particles as resonances at the $\mathrm{LHC}$ and laser beam experiments, Phys. Lett. B 795 (2019) 339 [arXiv:1903.04151] [INSPIRE].

[86] M. Bauer, M. Heiles, M. Neubert and A. Thamm, Axion-like particles at future colliders, Eur. Phys. J. C 79 (2019) 74 [arXiv: 1808.10323] [INSPIRE].

[87] CLIC study team, H. Braun et al., CLIC 2008 parameters, CERN-OPEN-2008-021, CERN, Geneva, Switzerland (2008) [CLIC-NOTE-764] [INSPIRE].

[88] CLIC and CLICDP collaborations, Updated baseline for a staged Compact Linear Collider, CERN-2016-004, CERN, Geneva, Switzerland (2016) [arXiv: 1608.07537] [InSPIRE].

[89] D. Dannheim et al., CLIC $e^{+} e^{-}$linear collider studies, arXiv:1208.1402 [INSPIRE].

[90] L. Linssen et al., Physics and detectors at CLIC: CLIC conceptual design report, arXiv: 1202.5940 [INSPIRE].

[91] R. Franceschini et al., The CLIC potential for new physics, CERN yellow report: monographs, volume 3, CERN-2018-009-M, CERN, Geneva, Switzerland (2018) [arXiv: 1812.02093] [INSPIRE].

[92] R. Franceschini, Beyond the Standard Model physics at CLIC, arXiv:1902.10125 [INSPIRE].

[93] ATLAS collaboration, Evidence for light-by-light scattering in heavy-ion collisions with the ATLAS detector at the LHC, Nature Phys. 13 (2017) 852 [arXiv:1702.01625] [INSPIRE].

[94] CMS collaboration, Evidence for light-by-light scattering in ultraperipheral $\mathrm{Pb}$ - $\mathrm{Pb}$ collisions at $\sqrt{s_{\mathrm{NN}}}=5.02 \mathrm{TeV}$, Nucl. Phys. A 982 (2019) 791 [arXiv: 1808.03524] [INSPIRE].

[95] ATLAS collaboration, Observation of light-by-light scattering in ultraperipheral $\mathrm{Pb}+\mathrm{Pb}$ collisions with the ATLAS detector, Phys. Rev. Lett. 123 (2019) 052001

[arXiv: 1904.03536] [INSPIRE].

[96] R.O. Coelho, V.P. Gonçalves, D.E. Martins and M. Rangel, Exclusive and diffractive $\gamma \gamma$ production in $\mathrm{Pb}-\mathrm{Pb}$ collisions at the LHC, HE-LHC and FCC,

Eur. Phys. J. C 80 (2020) 488 [arXiv:2002.03902] [INSPIRE].

[97] R.O. Coelho, V.P. Goncalves, D.E. Martins and M.S. Rangel, Production of axionlike particles in $\mathrm{Pb}-\mathrm{Pb}$ collisions at the $\mathrm{LHC}, \mathrm{HE}-\mathrm{LHC}$ and $\mathrm{FCC}$ : a phenomenological analysis, Phys. Lett. B 806 (2020) 135512 [arXiv:2002.06027] [INSPIRE].

[98] S.C. Inan and A.V. Kisselev, Probe of the Randall-Sundrum-like model with the small curvature via light-by-light scattering at the LHC, Phys. Rev. D 100 (2019) 095004 [arXiv: 1902.08615] [INSPIRE].

[99] S. Atağ, S.C. İnan and İ. Şahin, Extra dimensions in photon-induced two lepton final states at the CERN-LHC, Phys. Rev. D 80 (2009) 075009 [arXiv:0904.2687] [InSPIRE].

[100] S. Atag, S.C. İnan and İ. Şahin, Extra dimensions in $\gamma \gamma \rightarrow \gamma \gamma$ process at the CERN-LHC, JHEP 09 (2010) 042 [arXiv: 1005.4792] [INSPIRE]. 
[101] G. Jikia and A. Tkabladze, Photon-photon scattering at the photon linear collider, Phys. Lett. B 323 (1994) 453 [hep-ph/9312228] [INSPIRE].

[102] G.J. Gounaris, P.I. Porfyriadis and F.M. Renard, Light by light scattering at high-energy: a tool to reveal new particles, Phys. Lett. B 452 (1999) 76 [Erratum ibid. 464 (1999) 350] [Erratum ibid. 513 (2001) 431] [hep-ph/9812378] [INSPIRE].

[103] G.J. Gounaris, P.I. Porfyriadis and F.M. Renard, The $\gamma \gamma \rightarrow \gamma \gamma$ process in the standard and SUSY models at high-energies, Eur. Phys. J. C 9 (1999) 673 [hep-ph/9902230] [INSPIRE].

[104] R.H. Milburn, Electron scattering by an intense polarized photon field, Phys. Rev. Lett. 10 (1963) 75 [inSPIRE].

[105] F.R. Arutyunian and V.A. Tumanian, The Compton effect on relativistic electrons and the possibilty of obtaining high energy beams, Phys. Lett. 4 (1963) 176 [INSPIRE].

[106] I.F. Ginzburg, G.L. Kotkin, V.G. Serbo and V.I. Telnov, Production of high-energy colliding $\gamma \gamma$ and $\gamma$ e beams with a High Luminosity at Vlepp accelerators, JETP Lett. 34 (1981) 491 [Pisma Zh. Eksp. Teor. Fiz. 34 (1981) 514] [InSPIRE].

[107] I.F. Ginzburg, G.L. Kotkin, V.G. Serbo and V.I. Telnov, Colliding $\gamma e$ and $\gamma \gamma$ beams based on the single pass accelerators (of Vlepp type), Nucl. Instrum. Meth. 205 (1983) 47 [INSPIRE].

[108] I.F. Ginzburg, G.L. Kotkin, S.L. Panfil, V.G. Serbo and V.I. Telnov, Colliding $\gamma e$ and $\gamma \gamma$ beams based on the single pass $e^{+} e^{-}$accelerators II. Polarization effects, monochromatization improvement, Nucl. Instrum. Meth. A 219 (1984) 5 [INSPIRE].

[109] G. Cowan, K. Cranmer, E. Gross and O. Vitells, Asymptotic formulae for likelihood-based tests of new physics, Eur. Phys. J. C 71 (2011) 1554 [Erratum ibid. 73 (2013) 2501] [arXiv: 1007.1727] [INSPIRE].

[110] C.F. von Weizsäcker, Radiation emitted in collisions of very fast electrons, Z. Phys. 88 (1934) 612 [INSPIRE].

[111] E.J. Williams, Nature of the high-energy particles of penetrating radiation and status of ionization and radiation formulae, Phys. Rev. 45 (1934) 729 [INSPIRE].

[112] H. Terazawa, Two photon processes for particle production at high-energies, Rev. Mod. Phys. 45 (1973) 615 [InSPIRE].

[113] V.M. Budnev, I.F. Ginzburg, G.V. Meledin and V.G. Serbo, The two photon particle production mechanism. Physical problems. Applications. Equivalent photon approximation, Phys. Rept. 15 (1975) 181 [inSPIRE].

[114] C. Carimalo, P. Kessler and J. Parisi, On the validity of the equivalent photon approximation for virtual photon-photon collisions, Phys. Rev. D 20 (1979) 1057 [INSPIRE].

[115] DELPHI collaboration, Study of tau-pair production in photon-photon collisions at LEP and limits on the anomalous electromagnetic moments of the tau lepton, Eur. Phys. J. C 35 (2004) 159 [hep-ex/0406010] [inSPIRE].

[116] G. Baur, K. Hencken, D. Trautmann, S. Sadovsky and Y. Kharlov, Coherent $\gamma \gamma$ and $\gamma A$ interactions in very peripheral collisions at relativistic ion colliders, Phys. Rept. 364 (2002) 359 [hep-ph/0112211] [INSPIRE].

[117] J.E. Cieza Montalvo and O.J.P. Éboli, Composite vector leptoquarks in $e^{+} e^{-}$, $\gamma e$ and $\gamma \gamma$ colliders, Phys. Rev. D 47 (1993) 837 [hep-ph/9208242] [INSPIRE]. 
[118] J.E. Montalvo, G.H. Ulloa and M.D. Tonasse, Doubly charged Higgs from e- $\gamma$ scattering in the 3-3-1 model, Eur. Phys. J. C 72 (2012) 2210 [arXiv:1205.3822] [InSPIRE].

[119] A.A. Billur and M. Köksal, Probe of the electromagnetic moments of the tau lepton in gamma-gamma collisions at the CLIC, Phys. Rev. D 89 (2014) 037301 [arXiv:1306.5620] [INSPIRE].

[120] S. Ata $ّ$ g and E. Gürkanl, Prediction for CP-violation via electric dipole moment of $\tau$ lepton in $\gamma \gamma \rightarrow \tau^{+} \tau^{-}$process at CLIC, JHEP 06 (2016) 118 [arXiv:1512.03640] [INSPIRE].

[121] A.A. Billur, M. Köksal and A. Gutiérrez-Rodríguez, Improved sensitivity on the electromagnetic dipole moments of the top quark in $\gamma \gamma, \gamma \gamma^{*}$ and $\gamma^{*} \gamma^{*}$ collisions at the CLIC, Phys. Rev. D 96 (2017) 056007 [arXiv:1702.03708] [InSPIRE].

[122] M. Köksal, A.A. Billur and A. Gutierrez-Rodriguez, Bounds on the electromagnetic dipole moments through the single top production at the CLIC, Adv. High Energy Phys. 2017 (2017) 6738409 [arXiv: 1602.05991] [InSPIRE].

[123] M. Köksal, A.A. Billur, A. Gutiérrez-Rodríguez and M.A. Hernández-Ruíz, Model-independent sensitivity estimates for the electromagnetic dipole moments of the $\tau$-lepton at the CLIC, Phys. Rev. D 98 (2018) 015017 [arXiv:1804.02373] [INSPIRE].

[124] Y. Özgüven, S.C. İnan, A.A. Billur, M. Köksal and M.K. Bahar, Search for the anomalous electromagnetic moments of tau lepton through electron-photon scattering at CLIC, Nucl. Phys. B 923 (2017) 475 [arXiv:1609.08348] [INSPIRE].

[125] D. Atwood, S. Bar-Shalom and A. Soni, Gauge boson-gauge boson scattering in theories with large extra dimensions, Phys. Rev. D 61 (2000) 054003 [hep-ph/9906400] [INSPIRE]. 